\title{
Non-perturbative improvement of quark mass renormalization in two-flavour lattice QCD
}

\section{\# $L P H A$ \\ Collaboration}

\author{
Patrick Fritzsch ${ }^{a, b}$, Jochen Heitger ${ }^{b}$ and Nazario Tantalo ${ }^{c, d}$ \\ a University of Southampton, School of Physics and Astronomy \\ Highfield, Southampton SO17 1BJ, United Kingdom \\ ${ }^{b}$ Westfälische Wilhelms-Universität Münster, Institut für Theoretische Physik \\ Wilhelm-Klemm-Straße 9, D-48149 Münster, Germany \\ c INFN, Sezione di Roma "Tor Vergata" \\ c/o Università di Roma "Tor Vergata", Dipartimento di Fisica, \\ Via della Ricerca Scientifica 1, I-00133 Rome, Italy \\ d Centro "Enrico Fermi" \\ c/o Compendio Viminale, I-00184 Rome, Italy \\ E-mail: p.fritzsch@soton.ac.uk, heitger@uni-muenster.de, \\ nazario.tantalo@roma2.infn.it
}

\begin{abstract}
We non-perturbatively determine the renormalization constant and the improvement coefficients relating the renormalized current and subtracted quark mass of (quenched) valence quarks propagating in a sea of $\mathrm{O}(a)$ improved two massless quarks. We employ the Schrödinger functional scheme and fix the physical extent of the box by working at a constant value of the renormalized coupling. Our calculation yields results which cover two regions of bare parameter space. One is the weak-coupling region suitable for volumes of about half a fermi. By making simulations in this region, quarks as heavy as the bottom can be propagated with the full relativistic QCD action and renormalization problems in HQET can be solved non-perturbatively by a matching to QCD in finite volume. The other region refers to the common parameter range in large-volume simulations of two-flavour lattice QCD, where our results have particular relevance for charm physics applications.
\end{abstract}

KEYwords: Nonperturbative Effects, Lattice QCD, Quark Masses and SM Parameters, Heavy Quark Physics. 


\section{Contents}

1. Introduction 1

2. Improvement conditions at constant physics 2

2.1 The PCAC relation and mass non-degenerate quarks 3

2.2 Schrödinger functional correlation functions 5

$\begin{array}{lll}2.3 & \text { Estimators for } b_{\mathrm{A}}-b_{\mathrm{P}}, b_{\mathrm{m}} \text { and } Z & 7\end{array}$

3. Numerical computation and results $\quad 9$

$\begin{array}{ll}3.1 \text { The constant physics condition } & 9\end{array}$

3.2 Results in the weak-coupling region of finite-volume QCD 11

$\begin{array}{lll}3.3 & \text { Results in the coupling region of large-volume simulations } & 18\end{array}$

4. Lines of constant physics in finite-volume QCD 21

$\begin{array}{ll}\text { 5. Conclusions } & 27\end{array}$

\section{Introduction}

Hadronic observables can be calculated with non-perturbative accuracy by recurring to lattice QCD. Among the shortcomings of all the lattice formulations of the continuum theory are cutoff effects, an unavoidable source of systematic errors that is particularly relevant in the case of flavoured quantities. On the one hand, cutoff effects can be quantified and removed by repeating the calculations with different lattice spacings and by extrapolating the results to the continuum limit. On the other hand, the bigger are the leading cutoff effects the harder will be the extrapolations and/or the computational effort required to perform simulations at smaller values of the lattice spacing.

There exist lattice formulations of the continuum theory carrying leading cutoff effects proportional to the square of the lattice spacing. $\mathrm{O}(a)$ improvement is achieved at the price of a high computational cost by working with lattice Dirac operators, solutions of the celebrated Ginsparg-Wilson relation, that preserve at fixed cutoff a lattice version of chiral symmetry (see ref. [1] for a recent review). Automatic $\mathrm{O}(a)$ improvement of some or all physical observables can be also achieved by introducing in the naive lattice fermion action a dimension-five operator preserving a non-singlet chiral symmetry while breaking at the same time the doubling symmetry and another symmetry of the continuum action, e.g. parity in the case of the twisted mass formulation [2].

In this paper we concentrate on the Wilson formulation of lattice QCD that is very well understood, provides a strictly local fermion action, preserves parity and all the vector 
symmetries of the continuum theory and where $\mathrm{O}(a)$ improvement is not automatic, but can be obtained by implementing Symanzik's program [3, 4, 5]. Furthermore, improvement can be restricted to on-shell quantities and correlation functions at physical distances without any loss [6].

In particular we calculate non-perturbatively with two massless dynamical flavours of sea quarks the coefficients $b_{\mathrm{m}}$ and $b_{\mathrm{A}}-b_{\mathrm{P}}$ and the renormalization constant $Z$ that are needed in order to renormalize (quenched) valence quark masses in the improved theory. The same quantities have been obtained in the quenched case in refs. [7, 8, 9], and here we closely follow the strategy devised in these papers. We employ the Schrödinger functional scheme $[10,11,12,13]$ and fix the physical extent of the box by working at a constant value of the renormalized coupling.

Our results span two regions of bare parameter space. The first is a weak coupling region corresponding to a fixed physical volume of about $0.5 \mathrm{fm}$. Simulations on such a small volume are required in order to study heavy-light hadronic systems on the lattice with quarks as heavy as the bottom. Indeed, given the computing resources available nowadays, b-quarks can be propagated with their full relativistic action and without introducing unacceptably high cutoff effects only in sufficiently small volumes. These simulations can then be used to match non-perturbatively HQET with QCD [14], and physical results can be obtained by calculating in the effective theory the running of the renormalization constants down to scales corresponding to sufficiently large physical volumes. Alternatively, finitevolume QCD results can be corrected by calculating in the full theory the finite-volume effects by means of a finite-size scaling technique [15]. These two strategies can be profitably combined as it has been done in the quenched theory [16].

The other region of parameter space of our results spans the common range of bare couplings used in large-volume simulations of two-flavour lattice QCD, where non-perturbative improvement has particular relevance for charm physics applications [17, 18, 19].

\section{Improvement conditions at constant physics}

The basic idea of on-shell improvement of a lattice field theory such as Wilson's formulation of lattice QCD as employed in the present paper consists in adding counterterms to the unimproved lattice action and fields such that the leading cutoff effects are cancelled in on-shell amplitudes. Having identified the cutoff effect at fixed $g_{0}$, where a continuum symmetry is broken, the coefficients of the different improvement terms need to be fixed by suitable improvement conditions. These conditions can either require pure lattice artifacts, i.e. combinations of observables that are known to approach zero in the continuum limit of the theory, to vanish, or even more generally the scaling of some renormalized quantity. This then defines the values of the improvement coefficients as a function of the lattice spacing $a$ or, equivalently, of the bare gauge coupling, $g_{0}^{2}=6 / \beta$.

In perturbation theory, where in principle lattice artifacts can be obtained from any renormalized quantity by subtracting its value in the continuum limit, the improvement coefficients are unique. Beyond perturbation theory, where one wants to determine the improvement coefficients by Monte Carlo calculations, they do not remain unique and 
depend on the choice of the condition. This in turn can be exploited in order to choose an improvement condition with a good sensitivity to the coefficient in question. In Wilson's lattice QCD, the resulting ambiguity is of $\mathrm{O}(a)$ in the coefficients and of $\mathrm{O}\left(a^{2}\right)$ in the physical observables after improvement. Extra care has to be exercised, however, if these ambiguities are large. A typical example of such a case, which has already been identified in the quenched approximation $[8,9]$, is the renormalization of the subtracted bare quark mass in the $\mathrm{O}(a)$ improved theory, which amounts to consider QCD with non-degenerate quark masses.

The authors of ref. [21] have discussed the details of improvement and renormalization of the quark field bilinears in the presence of mass non-degenerate quarks. According to their results a flavour non-singlet bilinear $O^{i j}$ renormalizes as

$$
O_{\mathrm{R}}^{i j}=Z_{O}\left[1+b_{O} \frac{1}{2}\left(a m_{\mathrm{q}, i}+a m_{\mathrm{q}, j}\right)+\tilde{b}_{O} N_{\mathrm{f}} a m_{\mathrm{q}}^{(\text {sea })}\right] O^{i j},
$$

while, because of the presence of disconnected diagrams, flavour singlet bilinears require a different renormalization constant and additional improvement coefficients. In the rest of our paper we consider only flavour non-singlet quantities and work at vanishing sea quark masses so that we need neither to write down contributions proportional to the improvement coefficient $\tilde{b}_{O}$ nor to discuss renormalization of flavour singlet quantities any further.

Let us illustrate this now in more detail by directly entering the discussion of the situation in two-flavour QCD addressed by this work. To avoid notational overload and since any renormalization scale dependence is unimportant for the purpose of this section, we write $m_{\mathrm{R}}$ as shorthand for $\bar{m}(\mu)$ in the following.

\subsection{The PCAC relation and mass non-degenerate quarks}

For the formulation of proper improvement conditions in a non-perturbative calculation it is generally advantageous to use lattice artifacts that derive from a symmetry of the continuum field theory that is not respected by the lattice regularization. A particularly suited choice for the purpose of this work are violations of the PCAC relation between the axial vector current and the pseudo-scalar density. Assuming that on-shell $\mathrm{O}(a)$ improvement has been fully implemented and using renormalized currents in the lattice regularized theory, it reads

$$
\left\langle\widetilde{\partial}_{\mu}\left(A_{\mathrm{R}}\right)_{\mu}^{a}(x) \mathcal{O}\right\rangle=2 m_{\mathrm{R}}\left\langle\left(P_{\mathrm{R}}\right)^{a}(x) \mathcal{O}\right\rangle, \quad \widetilde{\partial}_{\mu}=\frac{1}{2}\left(\partial_{\mu}+\partial_{\mu}^{*}\right),
$$

up to $\mathrm{O}\left(a^{2}\right)$ corrections, as long as $x$ is not in the support of the source operator $\mathcal{O}$. We adopt the standard renormalization pattern of improved lattice currents in a mass independent scheme which is conventionally decomposed as

$$
m_{\mathrm{R}}=\frac{Z_{\mathrm{A}}\left(1+b_{\mathrm{A}} a m_{\mathrm{q}}\right)}{Z_{\mathrm{P}}\left(1+b_{\mathrm{P}} a m_{\mathrm{q}}\right)} m+\mathrm{O}\left(a^{2}\right), \quad m=\frac{1}{2} \frac{\left\langle\widetilde{\partial}_{\mu}\left(A_{\mathrm{I}}\right)_{\mu}^{a}(x) \mathcal{O}\right\rangle}{\left\langle P^{a}(x) \mathcal{O}\right\rangle} .
$$

Here, $m$ denotes the bare current quark mass defined through some matrix element of the bare PCAC relation, where $\left(A_{\mathrm{I}}\right)_{\mu}^{a}=A_{\mu}^{a}+a c_{\mathrm{A}} \widetilde{\partial}_{\mu} P^{a}$ is the $\mathrm{O}(a)$ improved axial current and $c_{\mathrm{A}}\left(g_{0}\right)$ is taken to be the non-perturbative value of the two-flavour theory [20]. 
On the other hand, starting from the bare quark mass $m_{0}$ of the Lagrangian, we have an alternative representation of the renormalized quark mass in terms of the $\mathrm{O}(a)$ improved subtracted bare quark mass, $\widetilde{m}_{\mathrm{q}}$, viz.

$$
m_{\mathrm{R}}=Z_{\mathrm{m}} \widetilde{m}_{\mathrm{q}}, \quad \widetilde{m}_{\mathrm{q}}=m_{\mathrm{q}}\left(1+b_{\mathrm{m}} a m_{\mathrm{q}}\right), \quad m_{\mathrm{q}}=m_{0}-m_{\mathrm{c}}
$$

By equating eqs. (2.3) and (2.4) one obtains the bare current quark mass in terms of the subtracted bare quark mass:

$$
\begin{aligned}
m & =\frac{Z_{\mathrm{m}} Z_{\mathrm{P}}}{Z_{\mathrm{A}}} \frac{\left(1+b_{\mathrm{m}} a m_{\mathrm{q}}\right)\left(1+b_{\mathrm{P}} a m_{\mathrm{q}}\right)}{\left(1+b_{\mathrm{A}} a m_{\mathrm{q}}\right)} m_{\mathrm{q}}+\mathrm{O}\left(a^{2}\right) \\
& =\frac{Z_{\mathrm{m}} Z_{\mathrm{P}}}{Z_{\mathrm{A}}}\left[1+\left(b_{\mathrm{m}}+b_{\mathrm{P}}-b_{\mathrm{A}}\right) a m_{\mathrm{q}}+\mathrm{O}\left(\left[a m_{\mathrm{q}}\right]^{2}\right)\right] m_{\mathrm{q}}+\mathrm{O}\left(a^{2}\right) .
\end{aligned}
$$

The renormalization factor

$$
Z\left(\widetilde{g}_{0}^{2}\right) \equiv \frac{Z_{\mathrm{m}}\left(\widetilde{g}_{0}^{2}, a \mu\right) Z_{\mathrm{P}}\left(\widetilde{g}_{0}^{2}, a \mu\right)}{Z_{\mathrm{A}}\left(\widetilde{g}_{0}^{2}\right)}
$$

is finite, because the divergence of $Z_{\mathrm{m}}$ is canceled by that of $Z_{\mathrm{P}}$. Therefore, $Z$ is only a function of the improved bare coupling, $\widetilde{g}_{0}^{2}=g_{0}^{2}\left(1+b_{\mathrm{g}} a m_{\mathrm{q}}^{(\mathrm{sea})}\right)$, while the second factor in (2.5) is not. Our simulations of the two-flavour theory were performed at vanishing sea quark mass $\left(a m_{\mathrm{q}}^{(\text {sea })} \approx 0\right)$ and the valence quarks entering the bilinears are treated in the quenched approximation, hence $\widetilde{g}_{0}^{2}=g_{0}^{2}$ holds throughout. ${ }^{1}$ To keep notation short, we do not explicitly write out the $g_{0}^{2}$-dependence in our improvement coefficients and $Z$ from now on.

To disentangle $Z, b_{\mathrm{m}}$ and $b_{\mathrm{P}}-b_{\mathrm{A}}$ in eq. (2.6) from each other later on, one has to consider non-degenerate current quark masses, $m_{\mathrm{R}, i} \neq m_{\mathrm{R}, j}$. In this theory the renormalized, improved axial currents and densities are given by a straightforward generalization of what was written down earlier [7]. More precisely, since the presence of non-degenerate masses breaks flavour symmetry, our starting point now are the off-diagonal bilinear fields,

$$
A_{\mu}^{i j}(x)=\bar{\psi}_{i}(x) \gamma_{\mu} \gamma_{5} \psi_{j}(x), \quad \quad P^{i j}(x)=\bar{\psi}_{i}(x) \gamma_{5} \psi_{j}(x) .
$$

As above, the renormalized PCAC relation

$$
\left\langle\widetilde{\partial}_{\mu}\left(A_{\mathrm{R}}\right)_{\mu}^{i j}(x) \mathcal{O}\right\rangle=\left(m_{\mathrm{R}, i}+m_{\mathrm{R}, j}\right)\left\langle\left(P_{\mathrm{R}}\right)^{i j}(x) \mathcal{O}\right\rangle+\mathrm{O}\left(a^{2}\right)
$$

has to be understood as an operator identity, with

$$
\begin{aligned}
& \left(A_{\mathrm{R}}\right)^{i j}(x)=Z_{\mathrm{A}}\left(g_{0}^{2}\right)\left[1+b_{\mathrm{A}}\left(g_{0}\right) \frac{1}{2}\left(a m_{\mathrm{q}, i}+a m_{\mathrm{q}, j}\right)\right]\left(A_{\mathrm{I}}\right)_{\mu}^{i j}(x), \\
& \left(P_{\mathrm{R}}\right)^{i j}(x)=Z_{\mathrm{P}}\left(g_{0}^{2}, a \mu\right)\left[1+b_{\mathrm{P}}\left(g_{0}\right) \frac{1}{2}\left(a m_{\mathrm{q}, i}+a m_{\mathrm{q}, j}\right)\right] P^{i j}(x)
\end{aligned}
$$

and $\left(A_{\mathrm{I}}\right)_{\mu}^{i j}=A_{\mu}^{i j}+a c_{\mathrm{A}} \widetilde{\partial}_{\mu} P^{i j}$. The renormalization constants $Z_{\mathrm{A}}$ and $Z_{\mathrm{P}}$ involved are the same as those in the theory with two mass-degenerate quarks, and the $b$-coefficients now

\footnotetext{
${ }^{1}$ For a more general treatment without these simplifications, the reader may consult ref. [21].
} 
multiply cutoff effects proportional to the average $\frac{1}{2}\left(a m_{\mathrm{q}, i}+a m_{\mathrm{q}, j}\right)$ of the subtracted bare quark masses.

Correspondingly, also the subtracted quark masses of each flavour $i$ get separately improved, but share the renormalization factor of the degenerate case, viz.

$$
m_{\mathrm{R}, i}=Z_{\mathrm{m}} \widetilde{m}_{\mathrm{q}, i} \cdot \quad \widetilde{m}_{\mathrm{q}, i}=m_{\mathrm{q}, i}\left(1+b_{\mathrm{m}} a m_{\mathrm{q}, i}\right), \quad m_{\mathrm{q}, i}=m_{0, i}-m_{\mathrm{c}}
$$

and in analogy to eq. (2.5),

$$
m_{\mathrm{R}, i}=\frac{Z_{\mathrm{A}}}{Z_{\mathrm{P}}} Z m_{\mathrm{q}, i}\left(1+b_{\mathrm{m}} a m_{\mathrm{q}, i}\right), \quad Z=\frac{Z_{\mathrm{m}} Z_{\mathrm{P}}}{Z_{\mathrm{A}}} .
$$

By inserting the last equation into the PCAC relation (2.9), and by solving for the associated bare current quark masses, we arrive at the generalization of eqs. (2.5) and (2.6) for the case of mass non-degenerate quarks:

$$
\begin{aligned}
& \frac{\left\langle\left(\widetilde{\partial}_{0} A_{0}^{i j}+a c_{\mathrm{A}} \partial_{0}^{*} \partial_{0} P^{i j}\right) \mathcal{O}\right\rangle}{2\left\langle P^{i j} \mathcal{O}\right\rangle}= \\
& Z\left[\frac{1}{2}\left(m_{\mathrm{q}, i}+m_{\mathrm{q}, j}\right)+\frac{1}{2} b_{\mathrm{m}}\left(a m_{\mathrm{q}, i}^{2}+a m_{\mathrm{q}, j}^{2}\right)-\frac{1}{4}\left(b_{\mathrm{A}}-b_{\mathrm{P}}\right) a\left(m_{\mathrm{q}, i}+m_{\mathrm{q}, j}\right)^{2}\right]
\end{aligned}
$$

again up to terms of $\mathrm{O}\left(a^{2}\right)$. Moreover, we introduce a third renormalized mass defined as the mean value of two given ones,

$$
\frac{1}{2}\left(m_{\mathrm{R}, 1}+m_{\mathrm{R}, 2}\right)=Z_{\mathrm{m}}\left[\frac{1}{2}\left(m_{\mathrm{q}, 1}+m_{\mathrm{q}, 2}\right)+b_{\mathrm{m}} a \frac{1}{2}\left(m_{\mathrm{q}, 1}^{2}+m_{\mathrm{q}, 2}^{2}\right)\right],
$$

which differs from the one obtained by the renormalization of the mean $m_{0,3}=\frac{1}{2}\left(m_{0,1}+\right.$ $\left.m_{0,2}\right)$ taken at the level of bare quark masses,

$$
m_{\mathrm{R}, 3} \equiv Z_{\mathrm{m}} m_{\mathrm{q}, 3}\left(1+b_{\mathrm{m}} a m_{\mathrm{q}, 3}\right)=Z_{\mathrm{m}}\left[\frac{1}{2}\left(m_{\mathrm{q}, 1}+m_{\mathrm{q}, 2}\right)+b_{\mathrm{m}} a \frac{1}{4}\left(m_{\mathrm{q}, 1}+m_{\mathrm{q}, 2}\right)^{2}\right] .
$$

The difference of the quadratic terms in eq. (2.15) and (2.16) can be used to estimate $b_{\mathrm{m}}$. By building appropriate differences with non-degenerate quarks it is possible to construct estimators for the improvement coefficient $b_{\mathrm{m}}$ and $b_{\mathrm{A}}-b_{\mathrm{P}}$ separately.

In the following we show how one can extract $b_{\mathrm{A}}-b_{\mathrm{P}}, b_{\mathrm{m}}$ and $Z$ by applying eq. (2.14) with a few external operators resp. kinematical conditions [7, 8] (see also [22]).

\subsection{Schrödinger functional correlation functions}

Particularly in the context of the QCD Schrödinger functional (SF) and its lattice formulation $[10,11,12,13]$, the use of PCAC has proven to be very advantageous to solve improvement and renormalization problems in many situations, because there one has a large flexibility to choose appropriate improvement and renormalization conditions. Beyond that, one can compute, e.g., the improvement coefficients also for small values of the bare coupling $g_{0}$, thereby making contact with their perturbative expansions. An overview is given, for instance, in ref. [23]. 
If $\zeta$ and $\bar{\zeta}$ denote boundary quark and anti-quark fields at Euclidean time $x_{0}=0$ [24], the field products summed over the spatial volume,

$$
\mathcal{O}^{a}=\frac{a^{6}}{L^{3}} \sum_{\mathbf{u}, \mathbf{v}} \bar{\zeta}(\mathbf{u}) \gamma_{5} \frac{1}{2} \tau^{a} \zeta(\mathbf{v}), \quad \mathcal{O}^{\prime a}=\frac{a^{6}}{L^{3}} \sum_{\mathbf{u}, \mathbf{v}} \bar{\zeta}^{\prime}(\mathbf{u}) \gamma_{5} \frac{1}{2} \tau^{a} \zeta^{\prime}(\mathbf{v})
$$

yield pseudo-scalar boundary sources projected onto zero momentum. From these, the boundary-to-bulk resp. forward SF correlation functions in the pseudo-scalar channel are constructed from the axial current and density as

$$
f_{\mathrm{A}}\left(x_{0}\right)=-\frac{a^{3}}{2} \sum_{\mathbf{x}}\left\langle A_{0}^{a}(x) \mathcal{O}^{a}\right\rangle, \quad f_{\mathrm{P}}\left(x_{0}\right)=-\frac{a^{3}}{2} \sum_{\mathbf{x}}\left\langle P^{a}(x) \mathcal{O}^{a}\right\rangle
$$

The corresponding bulk-to-boundary resp. backward correlation functions, $g_{\mathrm{A}, \mathrm{P}}$, are related to the former by a time reflection and fall off with $T-x_{0}$ from their sources at $x_{0}=T$ as do the forward correlation functions with $x_{0}$ from $x_{0}=0$. In a vanishing background field, this time reflection symmetry can be utilized by taking their mean before building secondary quantities, in order to reduce the statistical noise. In the theory with mass non-degenerate quarks the bare correlation functions generalize to

$$
f_{\mathrm{A}}^{i j}\left(x_{0}\right)=-\frac{a^{3}}{2} \sum_{\mathbf{x}}\left\langle A_{0}^{i j}(x) \mathcal{O}^{j i}\right\rangle, \quad f_{\mathrm{P}}^{i j}\left(x_{0}\right)=-\frac{a^{3}}{2} \sum_{\mathbf{x}}\left\langle P^{i j}(x) \mathcal{O}^{j i}\right\rangle .
$$

We do not apply a summation convention here, and the boundary sources $\mathcal{O}^{j i}$ are formally composed by non-degenerate boundary quark and anti-quark fields in the same way as the currents in eq. (2.8). Backward correlation functions $g_{\mathrm{A}, \mathrm{P}}^{i j}\left(T-x_{0}\right)$ are defined analogously.

Including the $\mathrm{O}(a)$ correction of the axial current as in eq. (2.10), the improved bare current quark masses ${ }^{2}$ expressed in terms of the just introduced SF correlation functions are given by

$$
m_{i j}\left(x_{0}\right) \equiv m_{i j}\left(x_{0} ; L / a, T / L, \theta\right)=\frac{\widetilde{\partial}_{0} f_{\mathrm{A}}^{i j}\left(x_{0}\right)+a c_{\mathrm{A}} \partial_{0}^{*} \partial_{0} f_{\mathrm{P}}^{i j}\left(x_{0}\right)}{2 f_{\mathrm{P}}^{i j}\left(x_{0}\right)},
$$

where, only here, we explicitly indicate their additional dependence on $L / a, T / L$ and the periodicity angle $\theta$ in the boundary conditions of the fermion fields. In the degenerate case $(i=j), m_{i j}$ reduces to the non-singlet PCAC mass of a flavour degenerate doublet. In this definition, $\widetilde{\partial}_{0}$ denotes the average of the usual forward and backward derivatives but, as in $[7,8,9]$, we have also employed current quark masses involving improved derivatives, obtained by the replacements

$$
\widetilde{\partial}_{0} \rightarrow \widetilde{\partial}_{0}\left(1-\frac{1}{6} a^{2} \partial_{0}^{*} \partial_{0}\right), \quad \partial_{0}^{*} \partial_{0} \rightarrow \partial_{0}^{*} \partial_{0}\left(1-\frac{1}{12} a^{2} \partial_{0}^{*} \partial_{0}\right)
$$

in order to eventually estimate $b_{\mathrm{m}}, b_{\mathrm{A}}-b_{\mathrm{P}}$ and $Z$. When acting on smooth functions, these derivatives have an error of $\mathrm{O}\left(g_{0}^{2} a^{2}, a^{4}\right)$.

\footnotetext{
${ }^{2}$ This expression for the PCAC masses is only $\mathrm{O}(a)$ improved up to a factor $1+\frac{1}{2}\left(b_{\mathrm{A}}-b_{\mathrm{P}}\right)\left(a m_{\mathrm{q}, i}+a m_{\mathrm{q}, j}\right)$ for quark mass dependent cutoff effects.
} 


\subsection{Estimators for $b_{\mathrm{A}}-b_{\mathrm{P}}, b_{\mathrm{m}}$ and $Z$}

Following the strategy already proposed for the quenched computation in $[8,9]$, we form ratios of suitable combinations of degenerate and non-degenerate current quark masses. Representing eq. (2.14) through the $m_{i j}$ now, it turns into

$$
m_{i j}=Z\left[\frac{1}{2}\left(m_{\mathrm{q}, i}+m_{\mathrm{q}, j}\right)+\frac{1}{2} b_{\mathrm{m}}\left(a m_{\mathrm{q}, i}^{2}+a m_{\mathrm{q}, j}^{2}\right)-\frac{1}{4}\left(b_{\mathrm{A}}-b_{\mathrm{P}}\right) a\left(m_{\mathrm{q}, i}+m_{\mathrm{q}, j}\right)^{2}\right] .
$$

This enables us to derive direct estimators for $b_{\mathrm{A}}-b_{\mathrm{P}}, b_{\mathrm{m}}$ and $Z$ in terms of various bare PCAC quark masses $m_{i j}$, which can by computed via SF correlation functions according to eq. (2.20). By exploiting the symmetry properties [8] of eq. (2.22) and keeping only the relevant terms, i.e.

$$
\begin{aligned}
2 a m_{12}-\left(a m_{11}+a m_{22}\right) & =Z \frac{1}{2}\left(b_{\mathrm{A}}-b_{\mathrm{P}}\right)\left(a m_{\mathrm{q}, 1}-m_{\mathrm{q}, 2}\right)^{2}\left[1+\mathrm{O}\left(a m_{\mathrm{q}, 1}+a m_{\mathrm{q}, 2}\right)\right] \\
a m_{11}-a m_{22} & =Z\left(a m_{\mathrm{q}, 1}-a m_{\mathrm{q}, 2}\right)\left[1+\mathrm{O}\left(a m_{\mathrm{q}, 1}+a m_{\mathrm{q}, 2}\right)\right] \\
a m_{12}-a m_{33} & =\frac{1}{4} b_{\mathrm{m}} Z\left(a m_{\mathrm{q}, 1}-a m_{\mathrm{q}, 2}\right)^{2}\left[1+\mathrm{O}\left(a m_{\mathrm{q}, 1}+a m_{\mathrm{q}, 2}\right)\right]
\end{aligned}
$$

it is straightforward to deduce the following ratios, which define estimators for the desired improvement coefficients and renormalization constant:

$$
\begin{aligned}
R_{\mathrm{AP}} & \equiv \frac{2\left(2 m_{12}-m_{11}-m_{22}\right)}{\left(m_{11}-m_{22}\right)\left(a m_{\mathrm{q}, 1}-a m_{\mathrm{q}, 2}\right)}=\left(b_{\mathrm{A}}-b_{\mathrm{P}}\right)\left[1+\mathrm{O}\left(a m_{\mathrm{q}, 1}+a m_{\mathrm{q}, 2}\right)\right] \\
R_{\mathrm{m}} & \equiv \frac{4\left(m_{12}-m_{33}\right)}{\left(m_{11}-m_{22}\right)\left(a m_{\mathrm{q}, 1}-a m_{\mathrm{q}, 2}\right)}=\quad b_{\mathrm{m}}\left[1+\mathrm{O}\left(a m_{\mathrm{q}, 1}+a m_{\mathrm{q}, 2}\right)\right] \\
R_{Z} & \equiv \frac{m_{11}-m_{22}}{m_{\mathrm{q}, 1}-m_{\mathrm{q}, 2}}+\left(R_{\mathrm{AP}}-R_{\mathrm{m}}\right)\left(a m_{11}+a m_{22}\right)=Z+\mathrm{O}\left(a^{2}\right)
\end{aligned}
$$

While the leading cutoff effects on the estimators for the improvement coefficients $R_{\mathrm{AP}}$ and $R_{\mathrm{m}}$ depend (linearly) on the bare subtracted quark masses, the leading correction to the estimator $R_{Z}$ for the renormalization factor is $\mathrm{O}\left(a^{2}\right)$ after inserting

$$
R_{\mathrm{AP}}-R_{\mathrm{m}} \equiv \frac{2\left(2 m_{33}-m_{11}-m_{22}\right)}{\left(m_{11}-m_{22}\right)\left(a m_{\mathrm{q}, 1}-a m_{\mathrm{q}, 2}\right)}=\left(b_{\mathrm{A}}-b_{\mathrm{P}}-b_{\mathrm{m}}\right)+\mathrm{O}\left(a m_{\mathrm{q}, 1}+a m_{\mathrm{q}, 2}\right) .
$$

The difference $R_{\mathrm{AP}}-R_{\mathrm{m}}$ does not depend on the off-diagonal mass $m_{12}$ and the cutoffdependence of $R_{Z}$ is the one expected for a renormalization constant in the $\mathrm{O}(a)$ improved theory. Note that the essential piece for the disentanglement of $R_{\mathrm{AP}}$ and $R_{\mathrm{m}}$ is the introduction of a third mass in accordance with

$$
m_{0,3}=\frac{1}{2}\left(m_{0,1}+m_{0,2}\right) \quad \Leftrightarrow \quad m_{\mathrm{q}, 3}=\frac{1}{2}\left(m_{\mathrm{q}, 1}+m_{\mathrm{q}, 2}\right)
$$

as already anticipated in eqs. (2.15) and (2.16) before.

Since the bare current quark masses $m_{i j}$ are functions of the time coordinate $x_{0}$, where the operator insertion of the currents $A_{0}$ and $P$ takes place, so do the estimators $R_{X}, X=\mathrm{AP}, \mathrm{m}, Z$, depend on $x_{0}$. Therefore, one has to specify the exact timeslice range used to compute the estimators $R_{X}$. This choice must be considered as being part 
of a particular improvement condition, which uniquely defines the ratios $R_{X}$ as smooth functions of $g_{0}$. Within the SF formalism, a good plateau in the local PCAC masses usually sets in around the midpoint of the time extension, $T / 2$. This need not necessarily be the case for observables derived from these masses, especially when investigating improvement coefficients which genuinely emerge only at order $a$. Fluctuations are in general expected to show up, depending on the quantity and the region in bare parameter space where the simulation takes place, but this does not invalidate the once chosen improvement condition.

As we will use $T=3 L / 2$ later on, our final estimators are defined as timeslice averages over the central third of the time extension:

$$
R_{X} \equiv \frac{a}{T-L / 2+a} \sum_{x_{0}=L / 2 a}^{(T-L / 2) / a} R_{X}\left(x_{0}\right), \quad X \in\{\mathrm{AP}, \mathrm{m}, Z\}
$$

Note that the main reason for this is rather to be less dependent on a special timeslice - which means to get a smoother and more reliable signal - than simply decreasing the size of the error. Furthermore, since the number of timeslices used for the plateau average scales with the lattice size, the physical size of this plateau is kept constant and hence automatically respects our requirement of a constant physics condition to be used below. subsectionGeneral remarks on improvement conditions at constant physics Before proceeding with the actual computation of $b_{\mathrm{A}}-b_{\mathrm{P}}, b_{\mathrm{m}}$ and $Z$, we would like to discuss the virtues of improvement conditions at constant physics on more general grounds [23].

In the aforementioned quenched investigations $[8,9]$ it was found that, e.g., the result for $b_{\mathrm{A}}-b_{\mathrm{P}}$ at $a \approx 0.1 \mathrm{fm}$ does depend significantly on the computational details, namely the timeslice location $x_{0}$ and the exact lattice representation of derivatives. (See also the upper right panel in figure 2 of the present work.) Obviously, this is quite an extreme example of an improvement coefficient that is difficult to determine, and its ambiguity from choosing a different improvement condition may even grow to the same order as the coefficient itself. The reason for such a behaviour lies in the fact that the $\mathrm{O}(a)$ effects are not dominating over the $\mathrm{O}\left(a^{2}\right)$ ones in the considered correlation functions. Presumably, the $\mathrm{O}(a)$ effects are just not very large.

One of the benefits of the Schrödinger functional pointed out before is that improvement conditions formulated through SF correlation functions can also be studied in perturbation theory. Choosing kinematical variables such as $x_{0} / L, T / L$ and $\theta$ exactly as in the non-perturbative setup, the expansion of an improvement coefficient, here denoted generically by $b$, is calculated as a series

$$
b\left(g_{0}, a / L\right) \sim b^{(0)}(a / L)+g_{0}^{2} b^{(1)}(a / L)+\mathrm{O}\left(g_{0}^{4}\right) .
$$

For our example of $b_{\mathrm{A}}-b_{\mathrm{P}}$ it was observed in ref. [8] that the perturbative coefficients $b^{(0)}$ and $b^{(1)}$ show a similar dependence on the kinematics as the non-perturbative results. Our statement on the uniqueness of the improvement coefficients in perturbation theory at the beginning of this section, however, means that although generically the functions $b^{(0)}(a / L)$ and $b^{(1)}(a / L)$ will depend on the kinematical choices made in the improvement condition, the values $b^{(0)}(0)$ and $b^{(1)}(0)$ do not and are unique. 
Hence, the following generic picture emerges. Once improvement conditions at constant physics are imposed, by which we mean that all length scales in the correlation functions that define the improvement condition are kept fixed in physical units, only the lattice spacing $a$ changes when $g_{0}$ is varied. This is just the situation, to which Symanzik's local effective theory of cutoff effects can be applied. As a consequence, improvement coefficients extracted from different constant physics conditions are then expected to rapidly approach an almost unique function of $g_{0}$ as $g_{0} \rightarrow 0$. While it is clear from this discussion that fixed $L$ in physical units is to be preferred whenever possible, in certain situations one may want to implement improvement conditions at fixed $L / a$ for practical reasons. But in such a setting, the $\mathrm{O}(a)$ ambiguities in the improvement coefficients would not go to zero as $g_{0} \rightarrow 0(a \rightarrow 0) .^{3}$

In the present situation, where we have to work at finite quark masses, also the combination $L m_{\mathrm{R}, i}$ should be kept constant. Note, however, that these constant physics conditions do not have to be satisfied very precisely, because this would affect corrections to an $\mathrm{O}(a)$ term only. In addition to the studies $[8,9]$ in quenched QCD, such conditions have meanwhile also been imposed for the determination of $c_{\mathrm{A}}[20]$ and $Z_{\mathrm{A}}[26]$ in the dynamical two-flavour theory.

Let us summarize the discussion of this subsection. While an unfortunately chosen improvement condition, but implemented at constant physics, may even enlarge the cutoff effects for intermediate $a$, it guarantees that linear $a$-effects are absent. Putting the improvement coefficient to zero or some perturbative approximation does not guarantee the latter, and the linear $a$-effects should at least be estimated in some way.

\section{Numerical computation and results}

The goal of this section is to non-perturbatively compute the improvement factors $b_{\mathrm{m}}\left(g_{0}\right)$ and $\left[b_{\mathrm{A}}-b_{\mathrm{P}}\right]\left(g_{0}\right)$ as well as the renormalization constant $Z\left(g_{0}\right)$ in the $N_{\mathrm{f}}=2$ dynamical case along the lines of the foregoing section.

\subsection{The constant physics condition}

In view of the freedom of possible legitimate choices for kinematical parameters within the SF formulation of lattice QCD [10], it was found to be advantageous to choose slightly different, particularly adapted settings in specifying a suitable constant physics condition for each of the parameter regions mentioned in the introduction.

In the $\mathrm{SF}$, the renormalization scale $\mu=1 / L$ and thus the physical volume are implicitly defined by demanding the non-perturbatively renormalized coupling to take a certain value. Therefore, to impose our improvement/normalization conditions on a line of constant physics, it is required to keep all length scales fixed as $g_{0}$ is varied, which we realize for the purposes of this work as

\footnotetext{
${ }^{3}$ E.g., improvement conditions at fixed $L / a$ were employed for the non-perturbative determination of $c_{\mathrm{sw}}$ and $c_{\mathrm{A}}$ in quenched QCD [25]. In this case, however, the small tree-level $a$-effect was subtracted from the non-perturbative ones to insure that the improvement coefficients go to their tree-level values exactly; furthermore, the conditions were chosen such that also $\left|b^{(1)}(a / L)-b^{(1)}(0)\right|$ is very small.
} 
- condition 1:

$$
\theta=0.5, \quad \bar{g}^{2}(L)=2.989 \quad \Leftrightarrow \quad L \approx 0.25 \mathrm{fm} \text { for } 6.2 \lesssim \beta \lesssim 6.6
$$

- condition 2:

$$
\theta=0, \quad L \approx 1.8 L^{*} \text { s.th. } \bar{g}^{2}\left(L^{*}\right)=5.5 \Leftrightarrow L \approx 1.2 \mathrm{fm} \text { for } 5.2 \leq \beta \leq 5.7
$$

supplemented by choosing as fixed kinematical parameters

$$
C_{k}=C_{k}^{\prime}=0 \text { (vanishing background field) , } \quad T / L=3 / 2,
$$

where $C_{k}$ and $C_{k}^{\prime}$ induce the Dirichlet boundary conditions of the gauge field at $x_{0}=0$ and $T$, respectively.

The first $\beta$-range ('condition 1') refers to a region of weak couplings encountered in solving renormalization problems of lattice HQET and in studies of B-physics observables in the continuum limit of finite-volume two-flavour lattice QCD, see refs. [27, 28] and section 4. Our second $\beta$-range ('condition 2') corresponds to lattice spacings of $0.09 \mathrm{fm} \gtrsim$ $a \gtrsim 0.04 \mathrm{fm}[29,30]$, which is the typical coupling region employed in $N_{\mathrm{f}}=2$ large-volume simulations. In this case, the choice $\theta=0$ (periodic boundary conditions) together with an intermediate physical volume of $L \approx 1.2 \mathrm{fm}$ is guided by experiences made in the context of the non-perturbative redetermination of the axial current renormalization constant $Z_{\mathrm{A}}$ in ref. [26]. There it was observed that this is a kinematical region, where $a^{2}$-effects (which can be significant at $a \approx 0.1 \mathrm{fm}$ ) are better suppressed, implying reduced intrinsic $a^{2}$-ambiguities in $Z_{\mathrm{A}}$, and $\mathrm{SF}$ simulations can be done close to or in the chiral limit of vanishing sea quark mass. The chiral limit can be reached in the simulations, when the infrared cutoff on the spectrum of the Dirac operator (in the SF dominantly controlled by $1 / T$ ) is sufficiently large, so that $T=3 L / 2 \approx 1.8 \mathrm{fm}$ is a feasible choice for this parameter region and the underlying $N_{\mathrm{f}}=2$ gauge field configurations could be shared among the two projects.

For the generation of our dynamical gauge field configurations with $N_{\mathrm{f}}=2$ massless sea quarks, we employed the HMC algorithm with two pseudo-fermion fields as proposed in [31, 32]. The particular implementation has been discussed and tested in refs. [33, 34] and following the latter, for the $\beta$-range and volume of (3.2) we always chose a molecular dynamics trajectory length of $\tau=2$, the only exception being $\beta=5.2$, where we set $\tau=1$. For the $\beta$-range and volume of (3.1), we had $\tau=1$ throughout. To prepare a light sea quark doublet of mass zero in the simulations, the corresponding hopping parameter, $\kappa_{1}$, was tuned to its critical value, $\kappa_{\mathrm{c}}$.

In order to complete the definition of the lines of constant physics, values for the bare PCAC masses of the valence quarks must be selected. In order to get an impression of the quark mass dependence in physical observables, we consider in analogy to the quenched study [9] two sets,

$$
\begin{array}{lll}
\text { set 1: } & L m_{11} \approx 0, & L m_{22} \approx 0.5, \\
\text { set 2: } & L m_{11} \approx 0, & L m_{22} \approx 2.5,
\end{array}
$$




\begin{tabular}{cccccc}
\hline$L / a$ & $T / a$ & $\beta$ & & $\kappa_{i}$ & \multicolumn{1}{c}{$L m_{i i}$} \\
\hline 10 & 15 & \multirow{2}{*}{6.1906} & $\kappa_{1}$ & 0.1360160 & $-0.0054(7)$ \\
& & & $\kappa_{2}$ & 0.1343182 & $+0.5004(6)$ \\
& & & $\widehat{\kappa}_{2}$ & 0.1276218 & $+2.5004(7)$ \\
12 & \multirow{2}{*}{18} & 6.3158 & $\kappa_{1}$ & 0.1357930 & $-0.0062(10)$ \\
& & & $\kappa_{2}$ & 0.1343782 & $+0.5008(9)$ \\
& & & $\widehat{\kappa}_{2}$ & 0.1287549 & $+2.5007(10)$ \\
16 & 24 & 6.5113 & $\kappa_{1}$ & 0.1354410 & $-0.0101(13)$ \\
& & & $\kappa_{2}$ & 0.1343872 & $+0.4949(13)$ \\
& & & $\widehat{\kappa}_{2}$ & 0.1301456 & $+2.4955(13)$ \\
20 & \multirow{2}{*}{30} & \multirow{2}{*}{6.6380} & $\kappa_{1}$ & 0.1351630 & $+0.0176(9)$ \\
& & & $\kappa_{2}$ & 0.1343562 & $+0.5024(9)$ \\
& & & $\widehat{\kappa}_{2}$ & 0.1309650 & $+2.5039(10)$ \\
\hline
\end{tabular}

Table 1: Results after tuning the hopping parameters in our small-volume computations to different values of the dimensionless (and plateau-averaged) PCAC masses $L m_{11}$ and $L m_{22}$ as specified in eqs. (3.4) and (3.5). Set 1 and set 2 share the same sea quark content, given by $\kappa_{1}=\kappa_{c}=\kappa_{1}$. The hopping parameters marked by a hat belong to set 2 .

where throughout this section all bare current quark masses are understood to be calculated according to an averaging prescription analogous to eq. (2.31). The appearing local current quark masses $m_{i i}\left(x_{0}\right)$ are evaluated using the standard lattice derivative as implied, following standard conventions, by its definition (2.20) based on the $\mathrm{O}(a)$ improved PCAC relation $(2.9)$.

The choice on $L m_{22}$ in set 1 is motivated by experiences from the quenched investigation, ref. [8], where it was argued to be advantageous w.r.t. the size of $\mathrm{O}(a)$ ambiguities encountered in perturbation theory. By contrast, the choice of $L m_{22} \approx 2.5$ in set 2 is closer to the typical b-quark region itself. The conditions on $L m_{22}$ have been satisfied by adjusting the hopping parameters $\kappa_{2}$, responsible for the mass value of the heavy valence quark flavour. The hopping parameter $\kappa_{3}$ needed to extract $b_{\mathrm{m}}$, which fixes a third mass value according to eq. (2.30), is given by $\kappa_{3}=2 \kappa_{1} \kappa_{2} /\left(\kappa_{1}+\kappa_{2}\right)$ and was directly implemented in the measurement code to avoid rounding errors. Furthermore, we exploit the additional time-reflection symmetry of the forward and backward boundary-to-bulk fermionic SF correlation functions introduced in the previous section by summing them in the analysis accordingly.

\subsection{Results in the weak-coupling region of finite-volume QCD}

The parameter values for calculating the ratios $R_{X}$ in $L \approx 0.25 \mathrm{fm}$ are given in table 1 . Our statistics consists of $200-300$ gauge field configurations stored at frequencies $5-10$ in units of molecular dynamics trajectories. The time extent $T=3 L / 2$ is chosen such that the plateau average, eq. (2.31), is less affected by finite-size effects stemming from the boundaries compared to the other natural choice $T=L$. After having produced gauge field configurations with $N_{\mathrm{f}}=2$ massless dynamical quarks, we performed one or two steps 

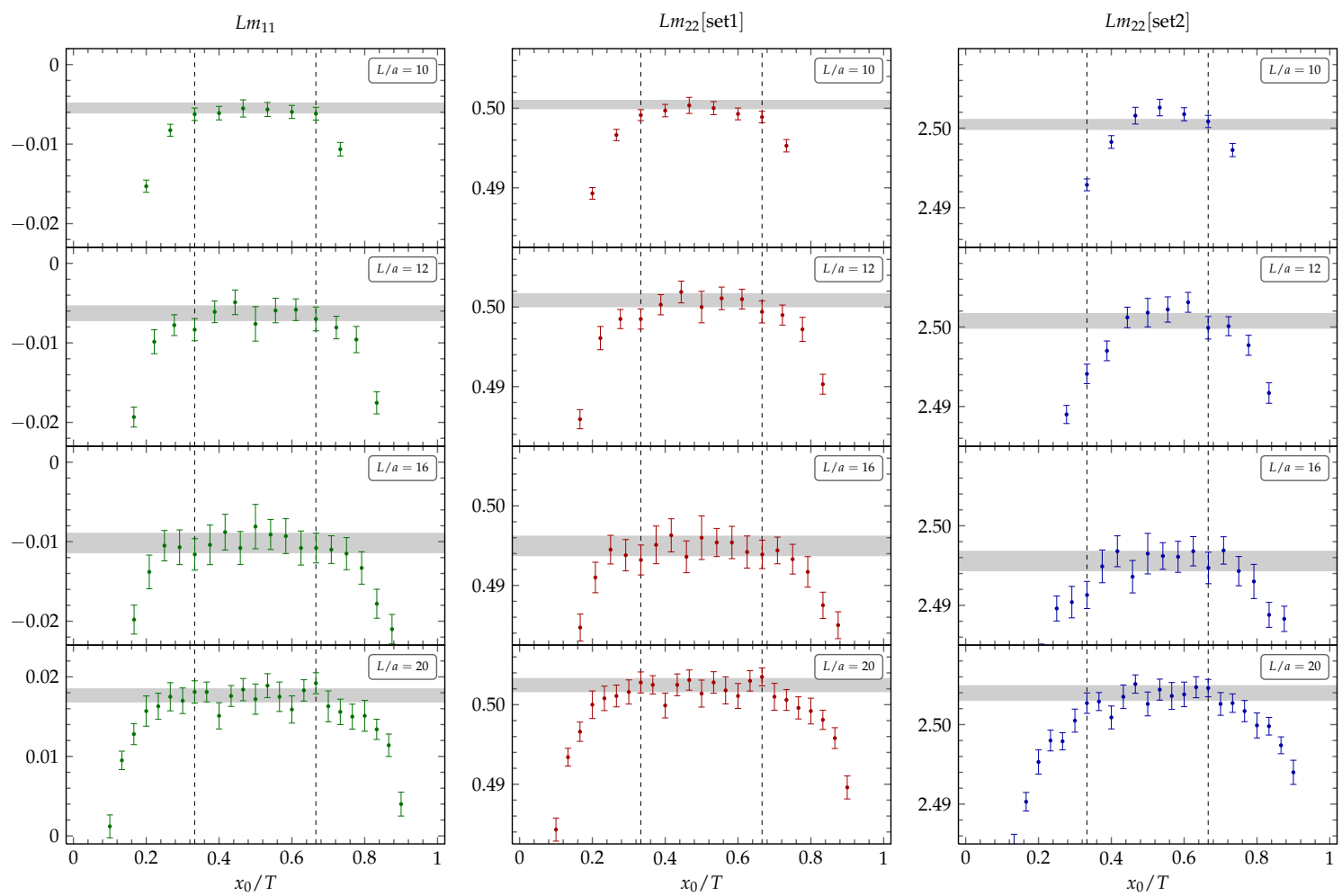

Figure 1: Time dependence of dimensionless PCAC masses obtained in small-volume QCD simulation after tuning the hopping parameters of the valence quarks as given in table 1 . The dashed lines define the plateau region used for the averaging as described in the text. Corresponding averaged mass values are shown as error bands in each subplot. $L m_{11} \approx 0$, and $L m_{22} \approx 0.5$ for set 1 resp. $L m_{22} \approx 2.5$ for set 2 are fulfilled within the desired accuracy.

of tuning to properly adjust $\kappa_{2}$ and satisfy the conditions ${ }^{4}$ imposed on $L m_{22}$ (see also figure 1). The estimators $R_{X}, X \in\{\mathrm{AP}, \mathrm{m}, Z\}$, are defined in terms of PCAC masses, which are secondary quantities themselves, built from SF correlation functions by means of eq. (2.20). For the evaluation of all our observables and their statistical errors we hence employed the $\Gamma$-method [35], which directly analyzes their autocorrelation functions. As a cross-check, a standard Jackknife procedure was applied as well. The results for $R_{X}$ from improvement condition 1 for set 1 and set 2 are listed in table 2 .

The two plots on the r.h.s. of figure 2 show the timeslice dependence $R_{\mathrm{AP}}\left(x_{0}\right)$ and $R_{\mathrm{m}}\left(x_{0}\right)$ for set 1 at $L / a=12$ and $\beta=6.3158$. The averaged estimators that represent our main results are shown as error bands. The filled symbols refer to improved time derivatives, (2.21), while the open symbols are obtained using the standard derivatives. Their difference can become quite large especially for $R_{\mathrm{AP}}$, as can be inferred from the plot. This is expected to be a pure cutoff effect and underlines the necessity to use improved derivatives. A plateau in $R_{\mathrm{AP}}\left(x_{0}\right)$ is visible in the data for both, standard and improved derivatives, but it is more pronounced for the latter.

\footnotetext{
${ }^{4}$ Similar to the situation in $[8,9]$, this is to a sufficient precision equivalent to keeping the corresponding renormalized masses $L Z_{\mathrm{A}} m / Z_{\mathrm{P}}$ fixed, as for the considered couplings the entering overall renormalization constant barely varies.
} 


\begin{tabular}{ccccccc}
\hline set & $L / a$ & $\beta$ & $b_{\mathrm{A}}-b_{\mathrm{P}}$ & $b_{\mathrm{m}}$ & $Z$ & $b_{\mathrm{A}}-b_{\mathrm{P}}-b_{\mathrm{m}}$ \\
\hline \multirow{2}{*}{1} & 10 & 6.1906 & $+0.0027(11)$ & $-0.6576(15)$ & $+1.10418(19)$ & $+0.6637(8)$ \\
& 12 & 6.3158 & $+0.0011(37)$ & $-0.6666(26)$ & $+1.10522(29)$ & $+0.6653(13)$ \\
& 16 & 6.5113 & $-0.006(11)$ & $-0.6637(36)$ & $+1.10395(29)$ & $+0.6614(22)$ \\
& 20 & 6.6380 & $-0.0050(29)$ & $-0.6636(48)$ & $+1.10333(23)$ & $+0.6648(21)$ \\
\hline \multirow{2}{*}{2} & 10 & 6.1906 & $+0.07261(41)$ & $-0.56417(38)$ & $+1.09522(11)$ & $+0.63820(37)$ \\
& 12 & 6.3158 & $+0.05177(61)$ & $-0.57800(66)$ & $+1.09747(13)$ & $+0.62971(60)$ \\
& 16 & 6.5113 & $+0.02950(60)$ & $-0.5955(10)$ & $+1.09945(13)$ & $+0.62617(69)$ \\
& 20 & 6.6380 & $+0.02101(68)$ & $-0.6077(11)$ & $+1.09997(11)$ & $+0.62914(71)$ \\
\hline
\end{tabular}

Table 2: Summary of results on the improvement coefficients and the renormalization factor under consideration in the volume with $L \approx 0.25 \mathrm{fm}$, for both sets of heavy quark masses (resp. constant physics conditions) using plateau-averaged masses and improved derivatives.

For a careful error analysis we also looked at the dependence of the mean values and errors on the number of points entering the plateau average about $x_{0}=T / 2$. This is exemplarily shown in the left plot of figure 2. There the leftmost point is the one at $x_{0}=T / 2$ in the plots on the right. In the examples given here, the plateau average is approached fast. This also nicely reflects that a plateau average is less affected by local fluctuations, which could distort the mean value by more than its associated uncertainty. If a plateau is visible, the averaging prescription - being considered as part of the definition of the $R_{X}$ as pointed out before - leads to a more reliable estimate for the mean value and the corresponding error.

The dependence of our main estimates $R_{X}$ is visualized in the left plot of figure 3 . The non-perturbative data points show a smooth dependence on $g_{0}^{2}$ as expected, suggesting that the correct scaling of discretization errors proportional to $a$ for the improvement coefficients and $a^{2}$ for the renormalization constant is met. Red data points correspond to our preferred choice, set 1 , whereas the blue points belong to set 2 . The qualitative dependence on $g_{0}^{2}$ is comparable to the quenched case [9]. Note, however, that such a direct comparison at the quantitative level is of limited meaning only, since in the latter case the bare parameter region of the simulations referred to a smaller physical volume and the relation between the lattice spacing and the bare coupling is different from the two-flavour theory.

In our fixed-volume computations, the lattice resolution gets smaller with decreasing $g_{0}^{2}$. Accordingly, the difference between both data sets decreases in this direction as it should be. Except for $R_{\mathrm{AP}}$, set 1, the non-perturbative points show a partly significant deviation from the one-loop perturbative predictions $[8,36]$ that are drawn as dashed lines in figure 3, too. For $R_{\mathrm{m}}$ this deviation is of $\mathrm{O}(10 \%)$ and for $R_{Z}$ still about $1 \%$. One also observes the results of set 2 to have a larger curvature, but a smaller error in the range of bare couplings considered here. This may be explained by the much larger valence quark mass, $\left.\left.L m_{22}\right|_{\text {set2 }} \approx 5 L m_{22}\right|_{\text {set1 }}$, where the dynamics is governed by the heavy quark flavour and dominates the fluctuations induced by the dynamical massless quarks in the 

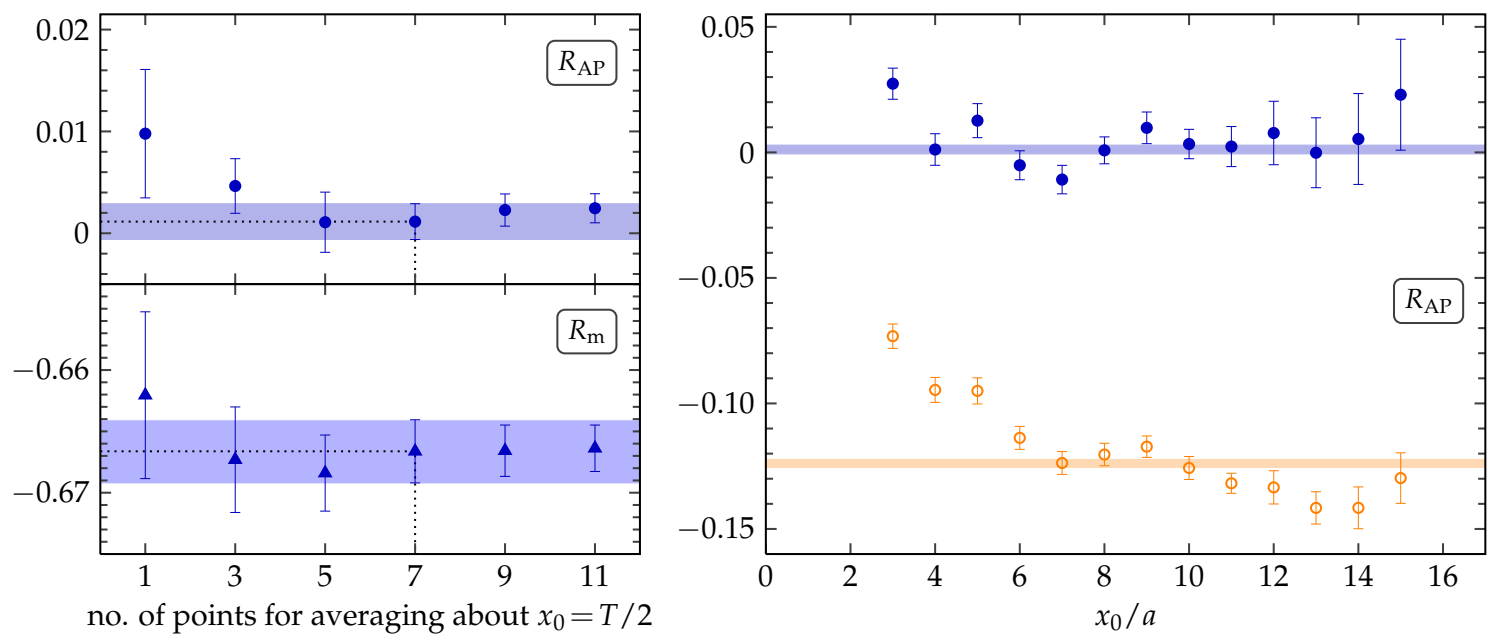

no. of points for averaging about $x_{0}=T / 2$

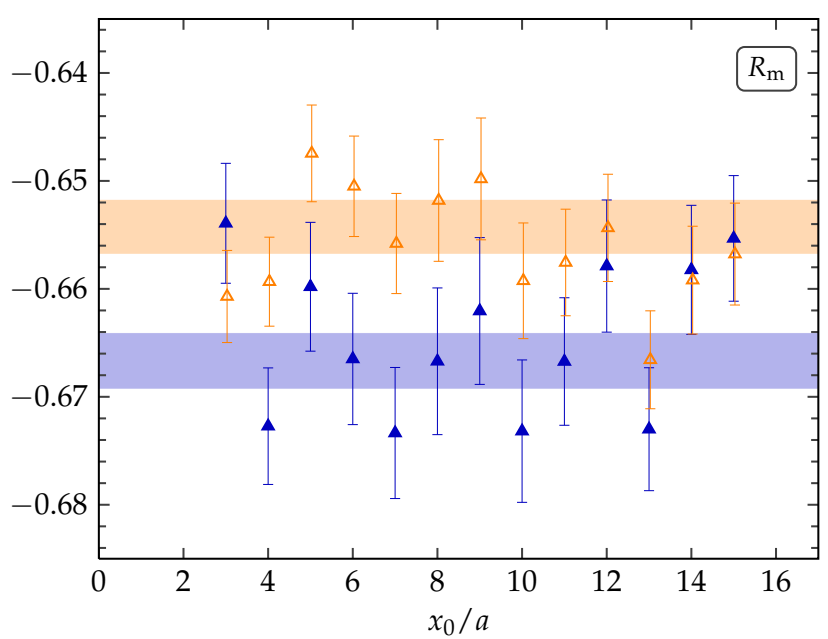

Figure 2: A typical data sample, showing $R_{\mathrm{AP}}$ and $R_{\mathrm{m}}$ data at $L / a=12$ and $\beta=6.3158$ for set 1 . Left: Dependence on the plateau size used for the averaging, with the preferred choice indicated by dotted lines. Right: Timeslice dependence of $R_{X}\left(x_{0}\right)$, if no plateau averaging of the local PCAC masses is applied. Open symbols denote results using standard derivatives, while filled symbols refer to those with improved lattice derivatives, eq. (2.21). For comparison, the shown (symmetric) error bands correspond to the results obtained from plateau-averaged masses.

gauge background.

The difference of $R_{X}$ between two improvement conditions is a measure for its $\mathrm{O}\left(a^{n}\right)$ ambiguity. In the extreme case of $b_{\mathrm{A}}-b_{\mathrm{P}}$, this difference in the results between sets 1 and 2 is of the same order as the improvement coefficient itself so that in such a situation it appears rather tempting to just put the coefficient to zero. In the light of the qualitative discussion in subsection 2.3, however, it should be obvious that it is then not guaranteed that linear $a$-effects are absent after improvement. Thus, an extrapolation to the continuum using an $\mathrm{O}\left(a^{2}\right)$ model function for the cutoff effects might then give significantly wrong results. Based on this warning, let us now study such ambiguities quantitatively. 

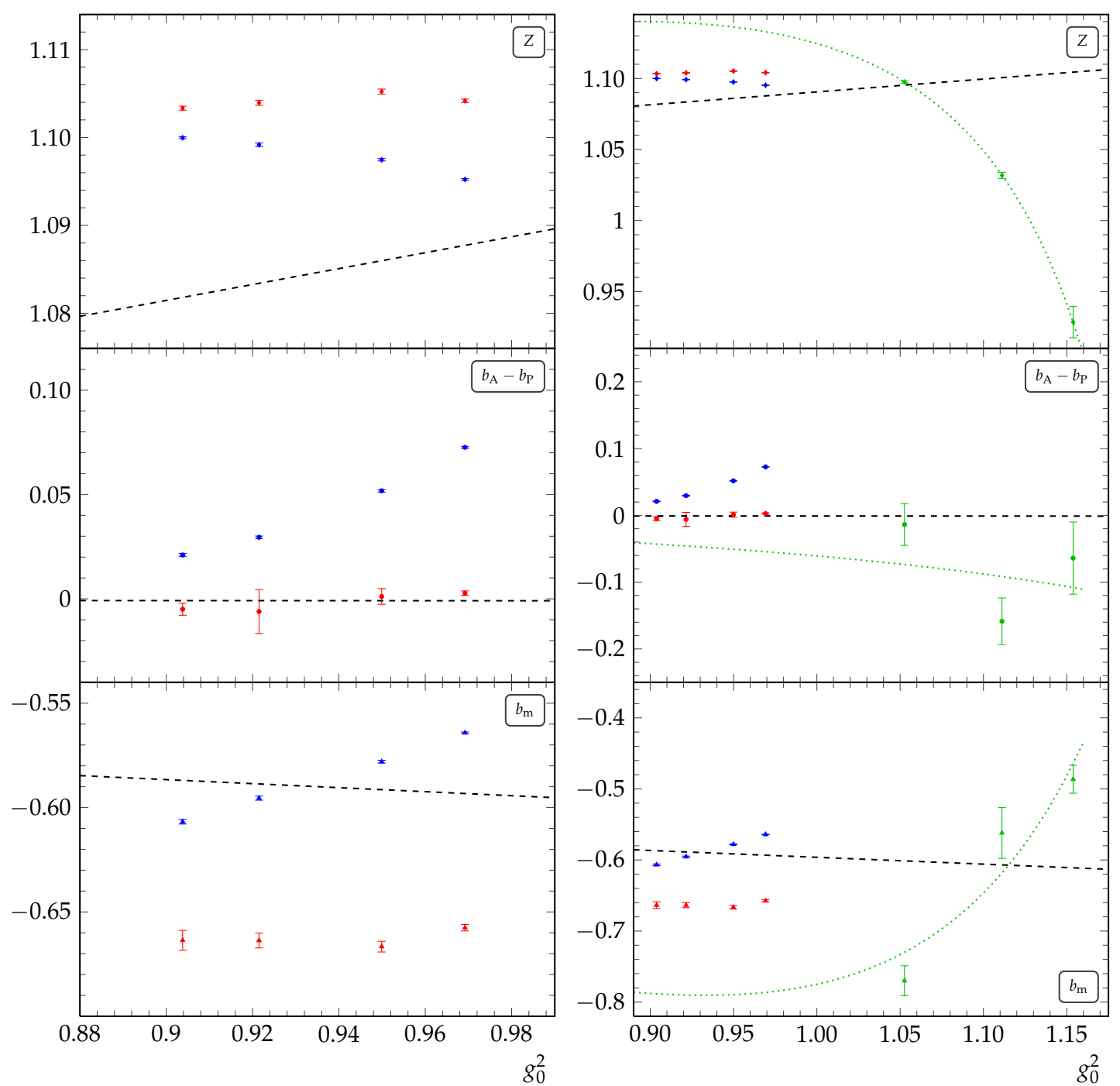

Figure 3: $g_{0}^{2}$-dependence of our final results for $Z, b_{\mathrm{A}}-b_{\mathrm{P}}$ and $b_{\mathrm{m}}$ from top to bottom. The dashed lines refer to the predictions from one-loop perturbation theory. Left: Results in the weak-coupling region of small-volume QCD (constant physics condition 1) for set 1 (red points) and set 2 (blue points). Right: Same points together with the corresponding results of subsection 3.3 in the region of larger couplings commonly employed in large-volume simulations (constant physics condition 2, green points).

\section{$\mathrm{O}\left(a^{n}\right)$ ambiguities}

As a consequence of the very idea of the constant physics condition, any other estimate $R_{X}$ (i.e. stemming from a different choice of improvement or renormalization condition) in general will yield a different functional dependence upon $g_{0}^{2}$ (and therefore $a / L$ ), but its differences are again smooth functions that must vanish in the continuum limit with a rate proportional to $a / L$ for improvement coefficients or $(a / L)^{2}$ for renormalization constants. Regardless of their actual size, these intrinsic $\mathrm{O}\left(a^{n}\right)$ ambiguities $(n=1,2)$ imply that rather than a numerical value at some given $\beta$, the important information lies in the correct $g_{0}^{2}$-dependence of the estimators $R_{X}, X \in\{\mathrm{AP}, \mathrm{m}, Z\}$, obtained by working at 

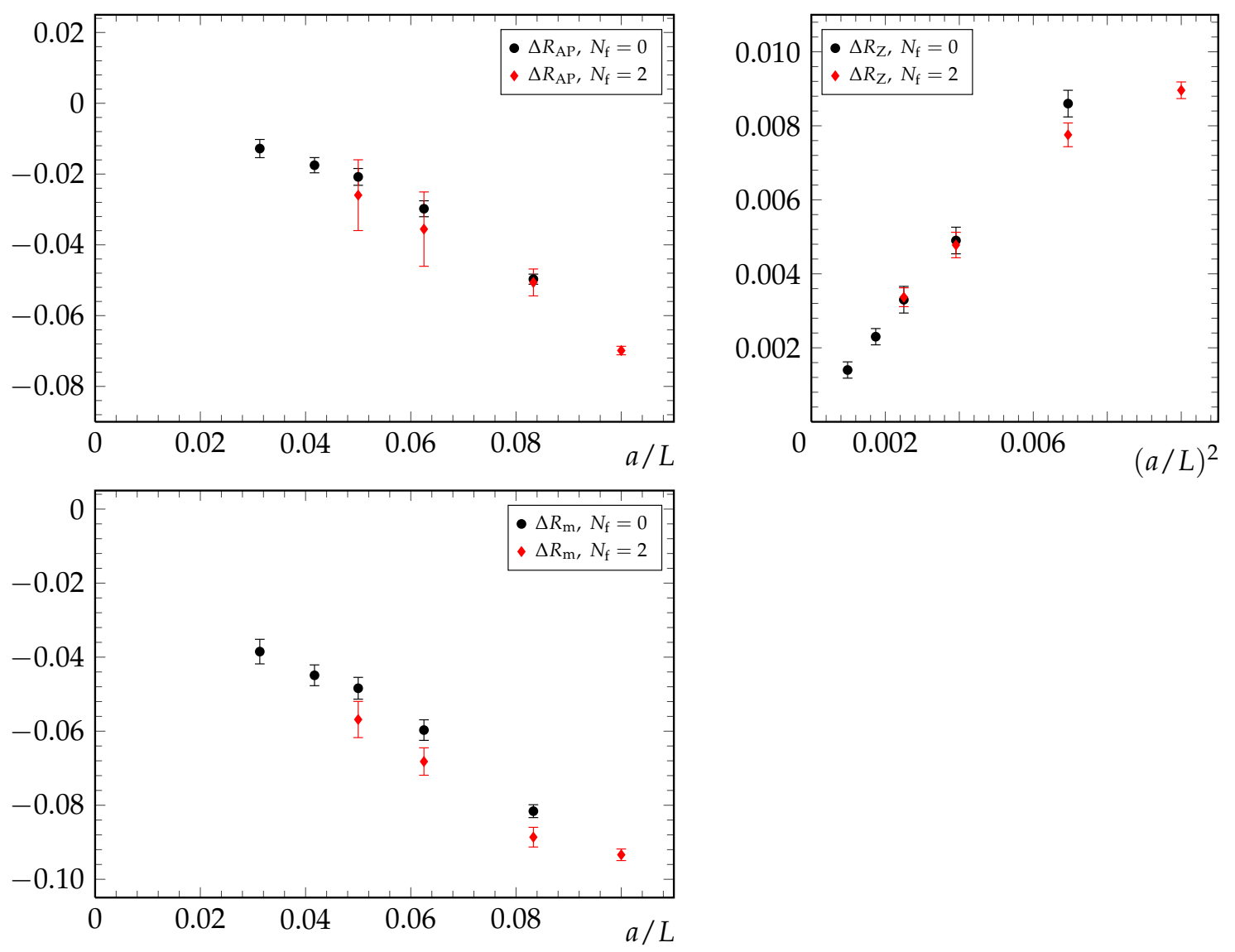

Figure 4: The differences $\Delta R_{X}$ of the estimators $R_{X}$ among the two sets of results, as defined in eq. (3.6). Within errors, the differences approach zero with a rate proportional to $a / L$ (for improvement coefficients) and $(a / L)^{2}$ (for the renormalization factor) as expected. The earlier quenched results [9] are added for comparison.

constant physics while varying $\beta$. To demonstrate this, we investigated a few alternative improvement conditions, which are either already provided by the two quark mass choices themselves, eqs. (3.4) and (3.5), or by defining the estimators $R_{X}$ with standard instead of improved derivatives.

Regarding the latter, we mentioned before that cutoff effects are visible between the use of standard and improved time derivatives, which are used to build the PCAC masses, and the reader may have wondered already about the large differences between these two choices of lattice derivatives in figure 2. But one should keep in mind that these results refer to an intermediate lattice spacing with $L / a=12$. When performing the full analysis for each data set with the standard derivatives, however, the differences to the results from employing improved derivatives were found to vanish in the limit $a \rightarrow 0$ as expected.

In addition, the two choices (3.4) and (3.5) made for the non-vanishing valence quark mass themselves correspond to different improvement conditions to extract the estimators $R_{X}$ and so may yield an impression of the intrinsic cutoff ambiguities. To this end we 


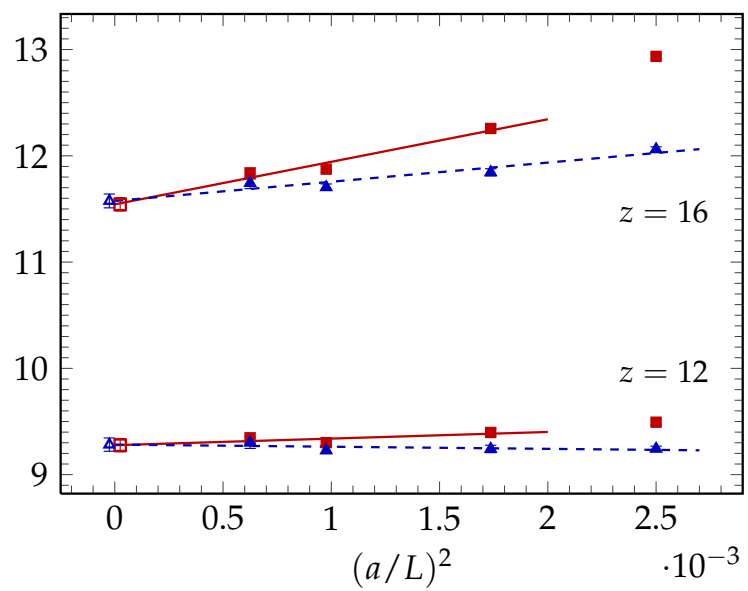

Figure 5: Unconstrained continuum extrapolations of $L_{1} \Gamma_{\mathrm{PS}}\left(z, g_{0}\right)$ for two fixed values of the renormalized quark, with different $b_{\mathrm{m}}$ and $Z$ entering the latter as described in the text. The data obtained via set 1 (red squares) and via set 2 (blue triangles) is fitted using three points (solid lines) and four points (dashed lines), respectively.

define

$$
\left.\Delta R_{X}\left(g_{0}\right) \equiv R_{X}\left(g_{0}\right)\right|_{\mathrm{set} 1}-\left.R_{X}\left(g_{0}\right)\right|_{\mathrm{set} 2}, \quad X \in\{\mathrm{AP}, \mathrm{m}, Z\} .
$$

From eqs. (2.26) and (2.27) one expects a linear $a$-dependence and according to (2.28), the cutoff dependence of $R_{Z}$ should be of $\mathrm{O}\left(a^{2}\right)$. For the values listed in table 2 we show the results for $\Delta R_{X}$ as red points (diamonds) in figure 4. While the difference $\Delta R_{Z}$ in the right panel clearly exhibits a linear approach towards zero, the $\mathrm{O}(a)$ ambiguities of $b_{\mathrm{A}}-b_{\mathrm{P}}$ and $b_{\mathrm{m}}$ reflected in the left panel are quite small as well, and their magnitudes rapidly decrease as $a / L \rightarrow 0$. The additional black points (circles) obtained in the quenched case [9] lie in the same ballpark, which can be attributed to the fact that the impact of the dynamical light quarks largely cancels out in the differences (3.6); on top of that, the cutoff ambiguities are dominated by the heavy quark mass content, which is about the same in both studies. In summary, the scaling behaviour observed in the various differences of estimators (3.6) confirms the theoretical expectation of the universality of the continuum limit and supports the reliability of the applied non-perturbative method also in the case of $N_{\mathrm{f}}=2$ dynamical quarks.

Although based on theoretical grounds discussed in subsection 2.3 we expect that physical observables involving sets of improvement coefficients from different constant physics conditions will have the same continuum limit, let us illustrate this in a concrete example. We consider the dimensionless combination $L_{1} \Gamma_{\mathrm{PS}}$, deriving from an effective energy that is extracted from the (improved) SF correlator of the time component of the axial vector current in a finite volume $L_{1}^{4}$ with $L_{1} \approx 0.5 \mathrm{fm}$,

$$
L_{1} \Gamma_{\mathrm{PS}}\left(z, g_{0}\right)=-\left.L_{1} \widetilde{\partial}_{0} \ln \left[f_{\mathrm{A}}\left(x_{0}, M\right)\right]\right|_{x_{0}=L_{1} / 2}, \quad z=L_{1} M
$$

We confront its lattice spacing dependence using $b_{\mathrm{m}}$ and $Z$ of set 1 with that using $b_{\mathrm{m}}$ and $Z$ of set 2 to fix the dimensionless renormalization group invariant (RGI) heavy quark mass 
to $z=12,16$ for lattice resolutions of $L_{1} / a=20-40$. For further technical details, see section 4. Both data sets and its unconstrained continuum extrapolations linear in $(a / L)^{2}$ are displayed in figure 5 and extrapolate to the same continuum limit. Associated statistical errors are smaller or of the order of the symbol size. The assumed quadratic scaling behaviour is clearly violated for the coarsest lattice at $z=16$ in set 1 though, because quark mass dependent cutoff effects are substantial in that case. Hence, we discarded the $L / a=20$ points in our fits of data set 1 . The fact that cutoff effects in $L_{1} \Gamma_{\mathrm{PS}}$ for both cases are larger for set 1 is not surprising, since the improvement condition of set 2 with $L_{0} m_{22} \approx 2.5$ and thus $L_{1} m_{22} \approx 5$ is much closer to the line in parameter space with $z=12,16$ along which $L_{1} \Gamma_{\mathrm{PS}}$ was computed. This general behaviour present in our new data was also observed in the quenched case [9]. The slope of the blue solid line at $z=16$ is approximately 6.5 times larger than the corresponding one at $z=12$. However, from the excellent agreement of the continuum limits in both cases one infers once more that our results correctly model the $g_{0}^{2}$-dependence of $b_{\mathrm{m}}$ and $Z$. No matter which set of improvement conditions is used, both entail convergence to the continuum limit with leading corrections of $\mathrm{O}\left(a^{2}\right)$ in the $\mathrm{O}(a)$ improved theory. Consequently we can conclude that the ambiguity introduced by choosing either set 1 or set 2 vanishes in the continuum limit also for other values of $z$ and/or observables and universality holds.

\subsection{Results in the coupling region of large-volume simulations}

Besides in the parameter range discussed in the previous subsection, which is relevant, for instance, in the context of the matching calculations of HQET and QCD and to which we will come back again in the next subsection, the estimators $R_{X}, X \in\{\mathrm{AP}, \mathrm{m}, Z\}$, are also of strong interest in the parameter region, where lattice computations of hadron masses and matrix elements in physically large volumes usually take place. This is particularly the case for mesons involving the charm quark, because large-volume studies in the quenched approximation $[17,18]$ have already given clear evidence for possibly substantial quark mass dependent cutoff effects down to $a \approx 0.05 \mathrm{fm}$ so that a non-perturbative removal of the associated $\mathrm{O}(a)$ corrections is highly desirable. Thus, we here provide $b_{\mathrm{A}}-b_{\mathrm{P}}, b_{\mathrm{m}}$ and $Z$ as functions in $g_{0}^{2}$ covering the range $5.2 \leq \beta \leq 5.7$, which then will allows to interpolate to the typical $\beta$-values relevant for large-volume computations in two-flavour lattice QCD.

In table 3 we summarize the corresponding simulation parameters, where the relation between $L^{*} / a$ and $g_{0}$ from ref. [30] was taken to translate the constant physics condition 2, eq. (3.2), to pairs of $(L / a, \beta)$. Owing to algorithmic instabilities caused by the appearance of very small, unphysical eigenvalues of the SF Wilson-Dirac operator [37, 38], the lattice with the coarsest lattice spacing (i.e. $L / a=12$ at $\beta=5.2$ ) could only be simulated down to bare PCAC sea quark masses of about $a m_{1} \equiv a m^{(\text {sea })} \approx 0.01$. Therefore, our computations for this parameter set were based on dynamical configurations at three sea quark mass values $\gtrsim 0.01$, made available to us through the determination of $Z_{\mathrm{A}}$ reported in [26], in order to extrapolate the results for $\beta=5.2$ to the chiral limit.

To eventually extract the estimators $R_{X}$ through eq. (3.2), we here considered the setting (3.4) for the non-degenerate valence quarks so that the heavier PCAC quark mass $L m_{22}$ had to be tuned to $\approx 0.5$, while the light one was again identified with the sea quark 


\begin{tabular}{cccccccc}
\hline$L / a$ & $T / a$ & $\beta$ & $\kappa_{1}$ & $L m_{11}$ & $\kappa_{2}$ & $L m_{22}$ & total \\
\hline 12 & 18 & 5.20 & 0.135500 & $0.2495(30)$ & 0.134677 & $0.5006(28)$ & 4000 \\
& & & 0.135700 & $0.1584(32)$ & 0.134601 & $0.4956(28)$ & 4000 \\
& & & 0.135800 & $0.1051(30)$ & 0.134512 & $0.5015(23)$ & 4000 \\
& & & $\kappa_{\mathrm{c}}$ & 0 & & $0.4986(36)$ & \\
\hline 16 & 24 & 5.40 & 0.136645 & $0.0094(26)$ & 0.135547 & $0.4996(20)$ & 750 \\
\hline 24 & 36 & 5.70 & 0.136704 & $0.0141(10)$ & 0.136000 & $0.5082(9)$ & 656 \\
\hline
\end{tabular}

Table 3: Simulation parameters to compute $b_{\mathrm{m}}$ and $b_{\mathrm{A}}-b_{\mathrm{P}}$ and $Z$ for a range of bare couplings typically covered in large-volume simulations of $N_{\mathrm{f}}=2$ lattice QCD. The rightmost column gives the number of decorrelated gauge field configurations used. The kinematical SF parameters are $(T / L, \theta)=(3 / 2,0)$, cf. eqs. (3.2) and (3.3).

\begin{tabular}{ccccccc}
\hline & $T / 3$ avr'd & $x_{0}=T / 2$ & $T / 3$ avr'd & $x_{0}=T / 2$ & $T / 3$ avr'd & $x_{0}=T / 2$ \\
\hline$\kappa_{1}$ & \multicolumn{2}{c}{0.135800} & \multicolumn{2}{c}{0.135700} & \multicolumn{2}{c}{0.135500} \\
$L m_{11}$ & $0.1051(30)$ & $0.1072(47)$ & $0.1584(32)$ & $0.1668(47)$ & $0.2495(30)$ & $0.2547(43)$ \\
$L m_{22}$ & $0.5015(23)$ & $0.5015(37)$ & $0.4956(28)$ & $0.5016(39)$ & $0.5006(28)$ & $0.5041(37)$ \\
\hline$b_{\mathrm{A}}-b_{\mathrm{P}}$ & $-0.098(50)$ & $-0.078(95)$ & $-0.040(42)$ & $-0.048(74)$ & $-0.086(25)$ & $-0.108(45)$ \\
$b_{\mathrm{m}}$ & $-0.453(28)$ & $-0.413(43)$ & $-0.513(25)$ & $-0.511(43)$ & $-0.485(20)$ & $-0.424(36)$ \\
$Z$ & $0.9262(23)$ & $0.9194(38)$ & $0.9305(21)$ & $0.9225(35)$ & $0.9191(16)$ & $0.9058(31)$ \\
\hline
\end{tabular}

Table 4: Results at $\beta=5.2$ before extrapolating to the massless limit, stemming from averages over the central $T / 3$ timeslices and from using $x_{0}=T / 2$ only.

mass, $L m_{11}=L m_{1}$, by setting $\kappa_{1}=\kappa_{1}\left(=\kappa_{c}\right.$ in case of $\beta=5.4,5.7$ only). $L m_{11}$ and $L m_{22}$ are also listed in table 3 .

For the $\beta=5.2$ data we do not observe a pronounced plateau in $R_{X}\left(x_{0}\right)$, and we thus gather in table 4 results stemming from our standard definition with an average taken over timeslices of the central third of the time extent, together with the corresponding results obtained at $x_{0}=T / 2$. Note that the improvement coefficients are well compatible within errors, whereas for the renormalization constant this is only the case for the smallest light quark mass. We hence decided to not alter our procedure of estimating these quantities using timeslice averages. As for the chiral extrapolation $L m_{11}=L m_{1} \rightarrow 0$ of the $\beta=5.2$ results, we investigated fits of $R_{X}$ to a constant as well as linear and quadratic fits using two or all three (light valence $=$ sea) quark mass points, which gave overall consistent results, since the dependence on the light quark mass is rather mild. Two- and three-point weighted averages turned out to be most conservative and as estimates in the massless limit we just quote the weighted averages of the numbers at the two lightest quark masses. To account for a systematic error due to $\mathrm{O}\left(a m_{1}\right)$ contaminations, we follow ref. [26] and inflate the error of the mean by its difference to the heaviest sea quark mass point linearly. 

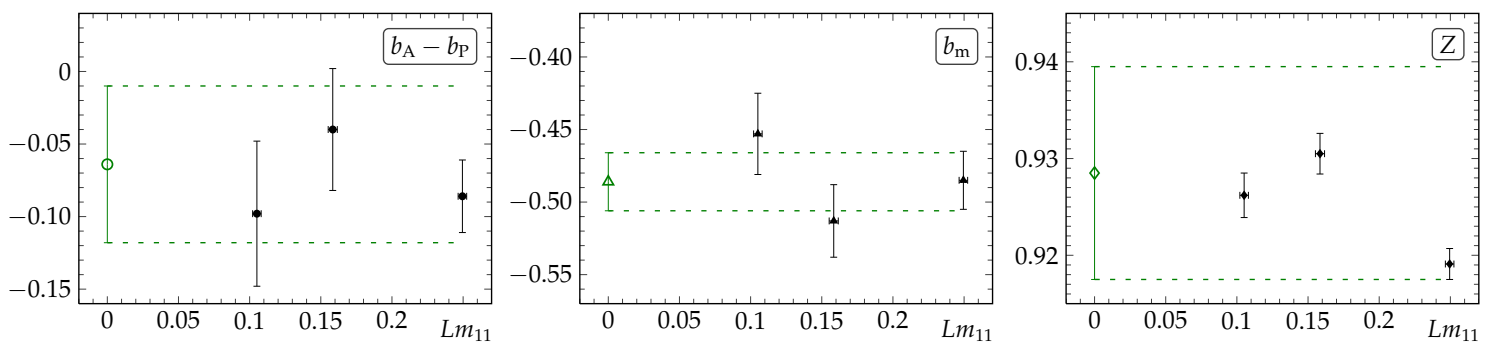

Figure 6: Extrapolations of the results at $\beta=5.2$ in table 4 to the chiral limit, $L m_{11}=L m_{1}=0$. The weighted two-point averages shown in the plot include our estimate of the $\mathrm{O}\left(a m_{1}\right)$ systematic uncertainty as explained in the text and quoted in table 5.

\begin{tabular}{cccccc}
\hline$L / a$ & $\beta$ & $\kappa_{1}$ & $b_{\mathrm{A}}-b_{\mathrm{P}}$ & $b_{\mathrm{m}}$ & $Z$ \\
\hline 12 & 5.20 & 0.135500 & $-0.086(25)$ & $-0.485(20)$ & $0.9191(16)$ \\
& & 0.135700 & $-0.040(42)$ & $-0.513(25)$ & $0.9305(21)$ \\
& & 0.135800 & $-0.098(50)$ & $-0.453(28)$ & $0.9262(23)$ \\
& & $\kappa_{\mathrm{c}}$ & $-0.064(32)(22)$ & $-0.486(19)(1)$ & $0.9285(16)(94)$ \\
\hline 16 & 5.40 & 0.136645 & $-0.159(37)$ & $-0.562(36)$ & $1.0319(22)$ \\
\hline 24 & 5.70 & 0.136704 & $-0.014(21)$ & $-0.770(21)$ & $1.0976(8)$ \\
\hline
\end{tabular}

Table 5: Results of $b_{\mathrm{A}}-b_{\mathrm{P}}, b_{\mathrm{m}}$ and $Z$ in the bare parameter region $5.2 \leq \beta \leq 5.7$. The quoted error on the results for $\beta=5.2$ is split into a statistical and a systematic one, the latter accounting for possible $\mathrm{O}\left(a m_{1}\right)$ contaminations from the extrapolation to the massless limit.

Our final results for all values of $\beta$ are summarized in table 5. Since simulations with massless dynamical quarks in this parameter region are numerically much more expensive, the $\beta=5.4$ and 5.7 data samples have a much smaller statistics compared to the ones reported in subsection 3.2. Consequently, the estimates in this parameter region have an uncertainty, which in general is one order of magnitude larger than in our small volume simulations at weaker couplings. Even though one might generically expect a universal curve connecting the results in distinct parameter regions, one encounters significantly different cutoff effects owing to different improvement conditions applied, as reflected on the r.h.s of figure 3 .

For later use of our results it seems appropriate to represent them as smooth functions in the interval $5.2 \lesssim \beta \lesssim 5.7$. As in the quenched computation [8], we can readily parametrize $Z, b_{\mathrm{A}}-b_{\mathrm{P}}$ and $b_{\mathrm{m}}$ by means of Padé fits in $g_{0}^{2}$ that are constrained to pass asymptotically into the perturbative one-loop predictions in the limit $g_{0}^{2} \rightarrow 0$. In case of the renormalization factor $Z$, the fit

$$
Z\left(g_{0}^{2}\right)=\left(1+0.090514 g_{0}^{2}\right) \times \frac{1-0.3922 g_{0}^{4}-0.2145 g_{0}^{6}}{1-0.6186 g_{0}^{4}}
$$

is found to describe our numbers very well, and the error to be attributed to this formula varies from about $1.2 \%$ at $\beta=5.2$ to less than $0.1 \%$ at $\beta=5.7$. Due to the scatter in the 
results for $b_{\mathrm{A}}-b_{\mathrm{P}}$, we quote as Padé fit parametrization

$$
\left[b_{\mathrm{A}}-b_{\mathrm{P}}\right]\left(g_{0}^{2}\right)=-0.00093 g_{0}^{2} \times \frac{1+23.59 g_{0}^{2}}{1-0.6235 g_{0}^{2}},
$$

which represents the data within two sigma of their overall uncertainty. Note that accidentally the parameter in the numerator is close to the quenched value, 23.306, and yet an unconstrained linear fit would represent the data points nearly equally well. Finally, for the improvement coefficient $b_{\mathrm{m}}$ we quote the interpolating formula

$$
b_{\mathrm{m}}\left(g_{0}^{2}\right)=\left(-0.5-0.09623 g_{0}^{2}\right) \times \frac{1-0.3737 g_{0}^{10}}{1-0.5181 g_{0}^{4}},
$$

still respecting the leading perturbative asymptotics. When using this formula at $g_{0}^{2}$-values different from $\beta=5.2,5.4,5.7$, one should attribute to $b_{\mathrm{m}}$ an uncertainty of the order of the error quoted in table 5 for the result at the nearest $\beta$.

\section{Lines of constant physics in finite-volume QCD}

As an immediate application of the results obtained in subsection 3.2, we address the computation of the dependence of heavy-light meson observables on the heavy quark mass in the continuum limit of finite-volume QCD. This is one of the key elements in the basic strategy [14] to non-perturbatively determine HQET parameters by a matching to QCD. Here, we only restrict the discussion to the fixing of the RGI heavy quark mass in a given finite volume to a series of values covering the b-quark mass down to the onset of the charm quark region, because it is this part, where $b_{\mathrm{m}}$ and $Z$ directly enter. A preliminary account on the subsequent computation of the heavy quark mass dependence of finite-volume QCD observables was given in the report [28].

Since we are eventually interested in quark mass dependent quantities evaluated in the continuum limit of finite-volume QCD, we want to calculate them for a series of bare parameters $\left(L / a, \beta, \kappa_{1}\right)$ such that the renormalized parameters in the light quark sector are fixed and in this way physics is kept constant along the approach to the continuum limit. As before, $\kappa_{\mathrm{l}}$ is the hopping parameter of the mass degenerate dynamical light quarks, while their PCAC mass is denoted by $m_{\mathrm{l}}$. In order to also fix the renormalized masses of the non-degenerate valence quarks in units of a physical scale later on, one first has to specify a suitable value of the renormalized SF coupling, $\bar{g}^{2}(L)$, which then, by its very definition [10], automatically fixes the spatial extent of the SF cylinder as the only scale of our system. Thus, in addition to $\theta=0.5$ and $T=L$, we choose

$$
\bar{g}^{2}\left(L_{0}\right)=2.989, \quad L_{0}=\frac{L_{1}}{2}, \quad L_{0} m_{1}\left(L_{0}\right)=0
$$

and thereby impose a constant physics condition on the renormalized coupling and the mass of the sea quark doublet in a finite volume of extent $L_{0}$. Implicitly, this condition defines the volume characterized by $L_{1}=2 L_{0}$, in which the non-perturbative matching between HQET and QCD will be performed [27]. Although an exact knowledge of $L_{1}$ in 


\begin{tabular}{cccclll}
\hline$L / a$ & $\beta$ & $Z_{\mathrm{P}}\left(g_{0}, L / a\right)$ & set & \multicolumn{1}{c}{$b_{\mathrm{A}}-b_{\mathrm{P}}$} & \multicolumn{1}{c}{$b_{\mathrm{m}}$} & \multicolumn{1}{c}{$Z$} \\
\hline \multirow{2}{10}{10} & 6.1569 & $0.6065(9)$ & 1 & $-0.0000(12)$ & $-0.6633(12)$ & $1.10443(17)$ \\
& & & 2 & $+0.07852(53)$ & $-0.56196(38)$ & $1.09488(13)$ \\
12 & 6.2483 & $0.5995(8)$ & 1 & $-0.0016(8)$ & $-0.6661(9)$ & $1.10475(12)$ \\
& & & 2 & $+0.06284(33)$ & $-0.57145(29)$ & $1.09632(8)$ \\
16 & 6.4574 & $0.5941(10)$ & 1 & $-0.0050(17)$ & $-0.6674(23)$ & $1.10455(17)$ \\
& & & 2 & $+0.03567(51)$ & $-0.59147(63)$ & $1.09888(10)$ \\
20 & \multirow{2}{*}{6.6380} & $0.5949(12)$ & 1 & $-0.0045(28)$ & $-0.6692(27)$ & $1.10379(17)$ \\
& & & 2 & $+0.02150(58)$ & $-0.60763(78)$ & $1.10021(10)$ \\
\hline
\end{tabular}

Table 6: Summary of the improvement coefficients and renormalization constants slightly extra/interpolated to ensure a fixed SF coupling in a volume with $L=L_{0} \approx 0.25 \mathrm{fm}$. The results at $L / a=20$ had not to be extrapolated. $Z_{\mathrm{P}}$ was determined independently following the nonperturbative renormalization procedure of [40].

physical units is not yet needed at this stage, one can infer from the known running of the SF coupling for $N_{\mathrm{f}}=2$, c.f. refs. [39, 30], that $L_{1}=2 L_{0} \approx 0.5 \mathrm{fm}$. Obviously, eq. (4.1) is identical to the corresponding settings of the constant physics 'condition 1', eq. (3.1), used for the determination of $b_{\mathrm{A}}-b_{\mathrm{P}}, b_{\mathrm{m}}$ and $Z$ themselves in subsection 3.2 and, in retrospect, explains this so far supposedly arbitrary choice.

The peculiar value $\bar{g}^{2}\left(L_{0}\right)=2.989$ was fixed by a simulation at $L_{0} / a=20, T=L_{0}$, in conjunction with tentative interpolations in $\beta=6 / g_{0}^{2}$ for given $L_{0} / a \leq 16$ to this target, based on the known dependence of the SF coupling and the current quark mass on the bare parameters $(\beta, \kappa)$ available from the data of ref. [39]..$^{5}$ The condition of zero light sea quark mass in eq. (4.1) is met by setting $\kappa_{\mathrm{l}}$ to the critical hopping parameter, $\kappa_{\mathrm{c}}$, estimated again on basis of published data [39], whereby a slight mismatch of $\left|L_{0} m_{1}\left(L_{0}\right)\right|<0.05$ of this condition is tolerable in practice. The triples $\left(L_{0} / a, \beta, \kappa_{1}\right)$ resulting from this procedure are those of table 1, used in our study of improvement and renormalization factors described above. In order to avoid a non-negligible systematic error from small violations of the condition (4.1) on results of any subsequent computations in $L_{1}^{4}$ though, the $\beta$-values were checked and fine-tuned further by additional simulations, which at the end led to the values in the second column of table 6 . The corresponding small shifts in $\beta$ affect our previous estimates of $b_{\mathrm{A}}-b_{\mathrm{P}}, b_{\mathrm{m}}$ and $Z$ only at a negligible level, because any deviation from the line of constant physics just entails a small change of the $\mathrm{O}\left(a^{2}\right)$ effects that we nevertheless account for by interpolations in $\beta$. The resulting, slightly moved values of $b_{\mathrm{A}}-b_{\mathrm{P}}, b_{\mathrm{m}}$ and $Z$ can be found in table 6 , too.

For the intended study of finite-volume QCD observables [28] we consider SF correlation functions composed of a non-degenerate heavy-light valence quark doublet, where the light valence quark mass is again chosen to be equal to the vanishing sea quark mass

\footnotetext{
${ }^{5}$ Using the known $\beta$-function and our experience from the quenched calculation [41], we can estimate that an uncertainty of about 0.04 in the coupling would translate via $L_{1}$ into an uncertainty in the b-quark mass of at most $0.5 \%$.
} 
adopted in the generation of the dynamical gauge configurations, i.e. it is set to $\kappa_{1}=\kappa_{\mathrm{c}}$. Computing the heavy quark mass dependence of observables then amounts to evaluate the correlators on these configurations in a volume $L_{1}^{3} \times T, T=L_{1}$, for a sequence of renormalized heavy valence quark mass values in a region extending from charm to beauty. For this purpose, the latter have to be fixed as precisely as possible for the same given pairs $\left(L_{0} / a, \beta\right)$ that already fix the volume $L_{1}$ via enforcing constant physics at $L_{0}=L_{1} / 2$ through eq. (4.1). Therefore, starting from (2.13), we exploit the $\mathrm{O}(a)$ improved relation between the subtracted bare heavy quark mass $m_{\mathrm{q}, \mathrm{h}}$ and the corresponding RGI mass, viz.

$$
M=h\left(L_{0}\right) Z_{\mathrm{m}}\left(g_{0}, L_{0} / a\right)\left(1+b_{\mathrm{m}}\left(g_{0}\right) a m_{\mathrm{q}, \mathrm{h}}\right) m_{\mathrm{q}, \mathrm{h}}+\mathrm{O}\left(a^{2}\right)
$$

where

$$
Z_{\mathrm{m}}\left(g_{0}, L_{0} / a\right)=\frac{Z\left(g_{0}\right) Z_{\mathrm{A}}\left(g_{0}\right)}{Z_{\mathrm{P}}\left(g_{0}, L_{0} / a\right)}, \quad a m_{\mathrm{q}, \mathrm{h}}=\frac{1}{2}\left(\frac{1}{\kappa_{\mathrm{h}}}-\frac{1}{\kappa_{\mathrm{c}}}\right)
$$

and the axial current renormalization constant $Z_{\mathrm{A}}$ in dependence of $g_{0}$ is non-perturbatively know for $N_{\mathrm{f}}=2$ from ref. [26]. The scale dependent renormalization constant $Z_{\mathrm{P}}$ was nonperturbatively determined in the course of our simulations in $L=L_{0}$, following exactly the prescription in ref. [40]. Its values relevant here (modulo a slight extrapolation in $\beta$ as in the case of $b_{\mathrm{A}}-b_{\mathrm{P}}, b_{\mathrm{m}}$ and $Z$ ) are listed in the third column of table 6. In eq. (4.2), there also appears the factor

$$
h\left(L_{0}\right) \equiv \frac{M}{\bar{m}\left(\mu_{0}\right)}=1.521(14), \quad \mu_{0}=\frac{1}{L_{0}}=\frac{2}{L_{1}},
$$

which represents the universal, regularization independent ratio of the RGI heavy quark mass, $M$, to the running quark mass $\bar{m}$ in the SF scheme at the renormalization scale $\mu_{0}$. $h\left(L_{0}\right)$ was evaluated by a reanalysis of available data on the non-perturbative quark mass renormalization in two-flavour QCD, also published in ref. [40].

Having all ingredients of eqs. (4.2) - (4.4) at hand, particularly the improvement coefficient $b_{\mathrm{m}}$ and the renormalization constant $Z$ obtained in this work, these equations can now straightforwardly be solved for any specific value of the dimensionless RGI heavy quark mass in $L_{1}$,

$$
z=L_{1} M
$$

to yield the desired hopping parameter associated with the mass of the heavy valence quark flavour,

$$
\kappa_{\mathrm{h}}=\kappa_{\mathrm{h}}\left(z, g_{0}\right)=\left\{\frac{1}{\kappa_{\mathrm{c}}}-\frac{1}{b_{\mathrm{m}}}\left(1-\sqrt{1+z \frac{4 b_{\mathrm{m}}}{\left[L_{1} / a\right] h Z_{\mathrm{m}}}}\right)\right\}^{-1},
$$

for which the heavy-light SF correlation functions and meson observables composed of them are to be evaluated. For $b_{\mathrm{m}}\left(g_{0}\right)$ and $Z\left(g_{0}\right)$ we employ the numbers belonging to the 'set 1' (cf. eq. (3.4)) of our results, because firstly, the $g_{0}^{2}$-dependence is weaker for $b_{\mathrm{m}}$ and secondly, with the setting $L_{0} m_{22} \approx 0.5$ in their determination (see eq. (3.4)) we rather meet 


\begin{tabular}{cccccc}
\hline & $z$ & \multicolumn{4}{c}{$L / a$} \\
\cline { 3 - 6 } & & 20 & 24 & 32 & 40 \\
\hline \multirow{2}{*}{$\beta$} & & 6.1569 & 6.2483 & 6.4574 & 6.6380 \\
$\kappa_{c}$ & - & 0.1360536 & 0.1359104 & 0.1355210 & 0.1351923 \\
$\kappa_{1}$ & 4 & 0.1327094 & 0.1331966 & 0.1335537 & 0.1336432 \\
$\kappa_{2}$ & 6 & 0.1309180 & 0.1317649 & 0.1325329 & 0.1328462 \\
$\kappa_{3}$ & 7 & 0.1299824 & 0.1310257 & 0.1320117 & 0.1324413 \\
$\kappa_{4}$ & 9 & 0.1280093 & 0.1294907 & 0.1309446 & 0.1316178 \\
$\kappa_{5}$ & 11 & 0.1258524 & 0.1278628 & 0.1298401 & 0.1307738 \\
$\kappa_{6}$ & 13 & 0.1234098 & 0.1261106 & 0.1286909 & 0.1299065 \\
$\kappa_{7}$ & 15 & 0.1204339 & 0.1241815 & 0.1274876 & 0.1290126 \\
$\kappa_{8}$ & 18 & - & 0.1206988 & 0.1255509 & 0.1276125 \\
$\kappa_{9}$ & 21 & - & 0.1140810 & 0.1233865 & 0.1261232 \\
\hline
\end{tabular}

Table 7: Hopping parameters $\kappa_{i} \equiv \kappa_{\mathrm{h}}^{(i)}$ from eq. (4.6), corresponding to fixed dimensionless RGI heavy quark masses $z=L M$ in a physical volume with $L=L_{1} \approx 0.5 \mathrm{fm}$ for different lattice resolutions.

the condition that in ref. [8] was found advantageous from the point of view of perturbation theory ${ }^{6}$. Owing to the sign and the order of magnitude of the non-perturbative values for $b_{\mathrm{m}}$ in the $\beta$-range relevant here, eq. (4.6) has no real solutions for arbitrarily high $z$-values. This demands to balance the upper bound on $z$ against the lattice resolutions one wants to simulate. In our case of $L_{1} / a=2 L_{0} / a=20,24,32,40$ we thus opted for nine values,

$$
z \in\{4,6,7,9,11,13,15,18,21\}
$$

which fairly span a range from around the charm to beyond the b-quark mass region. Equation (4.6) then yields the corresponding sets of heavy-flavour hopping parameters for each $L_{1} / a$ collected in table 7 , where solutions of (4.6) with $z=18,21$ only exist for inverse lattice spacings of $L_{1} / a=24,32,40$.

\section{Verification of $\mathrm{O}(a)$ improvement}

For fixing $z$ to the values in (4.7), one specific definition of the quark mass was applied, namely the relation between the bare subtracted heavy quark mass and the corresponding RGI mass (4.2), where the previously determined improvement coefficient $b_{\mathrm{m}}\left(g_{0}\right)$ and the renormalization constant $Z\left(g_{0}\right)$ enter. Nevertheless it appears tempting to have a look at equivalent definitions of the RGI heavy quark mass based on the PCAC relation, in order to provide another check of universality of the continuum limit in the $\mathrm{O}(a)$ improved theory.

\footnotetext{
${ }^{6}$ We recall in this context that it was explicitly demonstrated in subsection 3.2 that a typical observable such as the effective heavy-light meson energy approaches the same continuum limit, when computed at a fixed value of $z$ in which either $b_{\mathrm{m}}$ and $Z$ from 'set 1 ' or from 'set 2' enter.
} 


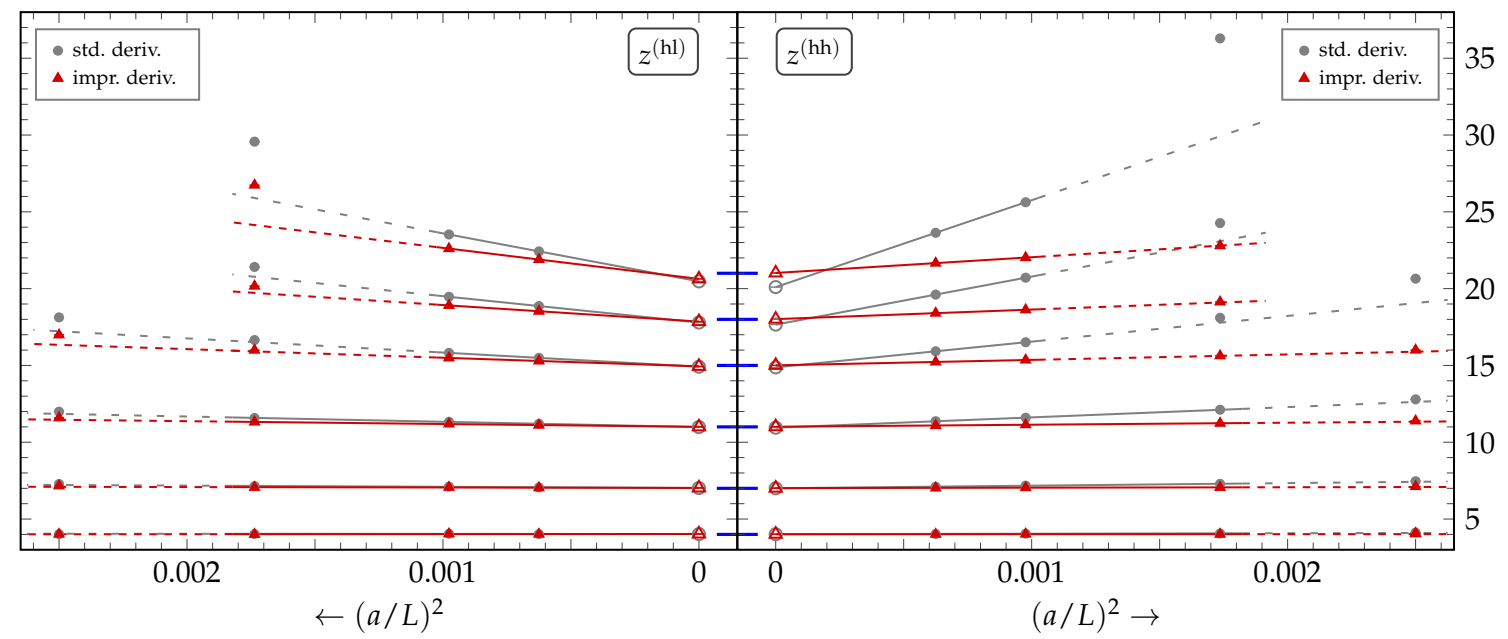

Figure 7: Continuum extrapolations of the dimensionless RGI PCAC heavy quark masses, defined through either the heavy-light (left) or the heavy-heavy (right) axial current, in a volume with $L=L_{1}$ for $\theta=0.5$. The statistical error is smaller than the symbol size. Dashed lines extend the fits to the points, which were not included in the extrapolation, and the bold ticks on the $z$-axis indicate the subset of $z$-values fixed via eq. (4.2) considered here.

As a spin-off, this allows to also apply our non-perturbative knowledge of $\left[b_{\mathrm{A}}-b_{\mathrm{P}}\right]\left(g_{0}\right)$ from subsection 3.2 in an actual calculation, even though the influence of the results on $b_{\mathrm{A}}-b_{\mathrm{P}}$ belonging to the 'set 1 ' in this parameter region is expected to be numerically very small. ${ }^{7}$

For each $\left(L_{1} / a, \beta, \kappa_{1}\right)$, we take the heavy valence quark hopping parameters corresponding to the subset $z \in\{4,7,11,15,18,21\}$ and compute the bare PCAC masses $m_{i j}$ of eq. (2.20) from the heavy-light $(i=\mathrm{h}, j=\mathrm{l})$ and heavy-heavy $(i=j=\mathrm{h})$ axial current and pseudo-scalar density. With eqs. (2.9) - (2.11) in mind, the renormalized PCAC quark masses then read

$$
\frac{1}{2}\left(m_{\mathrm{R}, i}+m_{\mathrm{R}, j}\right)=\frac{Z_{\mathrm{A}}\left(g_{0}\right)}{Z_{\mathrm{P}}\left(g_{0}, L_{0} / a\right)}\left\{1+\left[b_{\mathrm{A}}-b_{\mathrm{P}}\right]\left(g_{0}\right) \frac{1}{2}\left(a m_{\mathrm{q}, i}+a m_{\mathrm{q}, j}\right)\right\} m_{i j} .
$$

Since we are interested in recovering the above values of $z=L_{1} M$ by a continuum extrapolation of suitable PCAC quark masses, we need to express eq. (4.8) by RG invariants accordingly. Introducing the total renormalization factor $Z_{\mathrm{M}}\left(g_{0}\right)=h\left(L_{0}\right) Z_{\mathrm{A}}\left(g_{0}\right) / Z_{\mathrm{P}}\left(g_{0}, L_{0} / a\right)$, with $h\left(L_{0}\right)$ from eq. (4.4), we end up with

$$
\frac{1}{2}\left(z_{i}+z_{j}\right)=Z_{\mathrm{M}}\left\{1+\left[b_{\mathrm{A}}-b_{\mathrm{P}}\right] \frac{1}{2}\left(a m_{\mathrm{q}, i}+a m_{\mathrm{q}, j}\right)\right\} L_{1} m_{i j} .
$$

From the PCAC quark mass definition via the heavy-heavy current one thus directly has

$$
z^{(\mathrm{hh})}=Z_{\mathrm{M}}\left\{1+\left[b_{\mathrm{A}}-b_{\mathrm{P}}\right] a m_{\mathrm{q}, \mathrm{h}}\right\} L_{1} m_{\mathrm{hh}},
$$

while alternatively, $z$ can also by obtained through the quark mass definition via the heavylight current as

$$
z^{(\mathrm{hl})}=2 Z_{\mathrm{M}}\left\{1+\left[b_{\mathrm{A}}-b_{\mathrm{P}}\right] \frac{1}{2}\left(a m_{\mathrm{q}, \mathrm{h}}+a m_{\mathrm{q}, \mathrm{l}}\right)\right\} L_{1} m_{\mathrm{hl}}-z^{(11)} ;
$$

\footnotetext{
${ }^{7}$ Instead using the somewhat larger values of 'set 2' would not alter the outcome of this paragraph because $\Delta R_{\mathrm{AP}}$ vanishes in the continuum limit.
} 
here, $z^{(11)}$ as in eq. (4.10), but with $\mathrm{h} \rightarrow 1$. Since we have $\kappa_{\mathrm{l}}=\kappa_{\mathrm{c}}$ for our light sea and valence quarks, this reduces to

$$
z^{(\mathrm{hl})}=2 Z_{\mathrm{M}}\left\{1+\left[b_{\mathrm{A}}-b_{\mathrm{P}}\right] \frac{1}{2} a m_{\mathrm{q}, \mathrm{h}}\right\} L_{1} m_{\mathrm{hl}} .
$$

Based on universality arguments, we expect the continuum extrapolations of eqs. (4.10) and (4.12) to approach the corresponding values of $z$ fixed via the subtracted heavy quark mass, eq. (4.2). This is illustrated in figure 7. We show the results obtained from the heavy-light $\left(z^{(\mathrm{hl})}\right)$ and the heavy-heavy $\left(z^{(\mathrm{hh})}\right)$ current and compare the estimators for the PCAC masses using standard and improved lattice derivatives as introduced earlier, c.f. eq. (2.21). In qualitative agreement with our findings before, we in general observe that the cutoff effects increase with increasing values of $z$. The other expectation that cutoff effects are reduced when using improved lattice derivatives is clearly reflected as well. Whereas the continuum extrapolations for both lattice derivatives in the heavy-light case nicely match each other and coincide with the $z$-targets even at large values of $z$, one observes a slight mismatch for $z \geq 15$ in the heavy-heavy current case. This pattern can be explained by increasingly large quark mass dependent $a$-effects inherent to the standard lattice derivative. Since we used improved lattice derivatives in the course of estimating $b_{\mathrm{A}}-b_{\mathrm{P}}, b_{\mathrm{m}}$ and $Z$ underlying this analysis, it does not come as a surprise that the smallest cutoff effects are seen for $z^{(\mathrm{hh})}$ with improved derivatives. This is further supported by the fact that the extrapolating fit function meets the data points even beyond the range included in the fit.

\section{Error budget}

To summarize, $\left(L_{1} / a, \beta, \kappa_{\mathrm{l}}, \kappa_{i}\right)$, with $\kappa_{i} \equiv \kappa_{\mathrm{h}}^{(i)}$ and $i=1, \ldots, 9$, finally constitute the parameters of the numerical valence quark propagator calculations, from which the heavy-light correlation functions and the heavy quark mass dependence of the observables constructed from them are to be extracted. If for some reason (say, because of yet having used tentative results for the quantities entering (4.6)) a subsequent calculation was based on a marginally different set of $\kappa$-values, the associated small mismatch in having fixed $z$ for each $L_{1} / a$ can easily be corrected by a small interpolation.

At this point it still remains to quantify the uncertainty of $z$, which comes into play through the various factors and coefficients it depends on and which itself has to be propagated into any secondary quantity that is regarded as a function of $z$. With the estimate $\Delta Z_{\mathrm{A}} / Z_{\mathrm{A}} \approx 0.36 \%$ as quoted in [26] and $\Delta Z_{\mathrm{P}} / Z_{\mathrm{P}}, \Delta Z / Z$ as well as $\Delta b_{\mathrm{m}} / b_{\mathrm{m}}$ taken from table 6 , ones obtains according to the standard rules of Gaussian error propagation an accumulated relative error on $z$ in the range $0.38 \% \leq(\Delta z / z) \leq 0.41 \%$. This holds for all values of $z$ in (4.7) and inverse lattice resolutions $L_{1} / a$, but without the contribution from the continuum factor $h\left(L_{0}\right)$. The latter represents with $\Delta h / h=0.92 \%$ the dominating source of uncertainty in the total error budget of $\Delta z / z=1.01 \%$, when the fixing of $z$ proceeds as described here. 


\section{Conclusions}

In this paper we have non-perturbatively determined the renormalization constant and the improvement coefficients relating the renormalized current and subtracted quark mass in $\mathrm{O}(a)$ improved two-flavour lattice QCD. To solve this rather technical and difficult numerical problem, improvement and normalization conditions on lines of constant physics were imposed, which ensures a smooth dependence of these quantities on the bare coupling $g_{0}$ and the correct scaling of discretization errors proportional to $a$ (for the improvement coefficients) and $a^{2}$ (for the renormalization constant), respectively. In particular, our results open the way to a precision study of B-physics on the lattice, whose feasibility has already been demonstrated within the very useful test-laboratory of the quenched approximation over the last years [15, 16, 41, 42].

An important element within these approaches is the separation of the scales characterizing heavy-light systems and the introduction of small physical volumes in order to perform QCD calculations with relativistic b-quarks. These simulations can only be performed by knowing the renormalization constants and improvement coefficients computed in the present paper for the dynamical two-flavour theory. As explained in section 4, with the help of the latter the renormalization group invariant heavy quark mass in finite-volume simulations of the $\mathrm{O}(a)$ improved theory can accurately be fixed to values from the b- down to the c-quark region. Moreover, our results are also required when computing charmed observables by making simulations on the same volumes used to study light flavour physics.

In the $N_{\mathrm{f}}=2$ dynamical case, the programme of calculating the HQET parameters at $\mathrm{O}\left(1 / m_{\mathrm{b}}\right)$ non-perturbatively by a matching to $\mathrm{QCD}$, which already employs part of the results of this work, is well advanced and progressing fast and will allow to determine several phenomenologically interesting quantities such as, for instance, the b-quark mass and B-meson decay constant. As a further interesting direction for applications of the results of our work let us mention the non-perturbative tests of the HQET expansion in the spirit of the quenched investigation [43], which may greatly improve our confidence in the use of the effective theory. This will be presented in a forthcoming publication.

\section{Acknowledgments}

We are indebted to G. M. de Divitiis, M. Della Morte, H. Meyer, R. Petronzio, H. Simma, R. Sommer and S. Takeda for fruitful discussions and their contributions at an early stage of this work. We also would like to thank R. Sommer for a critical reading of the manuscript. This work is part of the ALPHA Collaboration research programme. We thank NIC/DESY and INFN for allocating computer time on the APE computers to this project as well as the staff of the computer centers at Zeuthen and Rome for their support. We acknowledge partial support by the European Community through EU Contract No. MRTN-CT-2006035482, "FLAVIAnet". P. F. and J. H. also acknowledge financial support by the Deutsche Forschungsgemeinschaft (DFG) under grant HE 4517/2-1. Some of the results presented here have already been published in [44]. 


\section{References}

[1] D. B. Kaplan, Chiral Symmetry and Lattice Fermions, 0912.2560.

[2] R. Frezzotti and G. C. Rossi, Chirally improving Wilson fermions. I: O(a) improvement, J. High Energy Phys. 08 (2004) 007 [hep-lat/0306014].

[3] K. Symanzik, Continuum limit and improved action in lattice theories: 1. Principles and $\phi^{4}$ theory, Nucl. Phys. B226 (1983) 187.

[4] K. Symanzik, Continuum limit and improved action in lattice theories: 2. O(N) non-linear sigma model in perturbation theory, Nucl. Phys. B226 (1983) 205.

[5] B. Sheikholeslami and R. Wohlert, Improved continuum limit lattice action for QCD with Wilson fermions, Nucl. Phys. B259 (1985) 572.

[6] M. Lüscher and P. Weisz, On-shell improved lattice gauge theories, Commun. Math. Phys. 97 (1985) 59. Erratum: ibid. 98 (1985) 433.

[7] G. M. de Divitiis and R. Petronzio, Non-perturbative renormalization constants on the lattice from flavor non-singlet ward identities, Phys. Lett. B419 (1998) 311 [hep-lat/9710071].

[8] ALPHA Collaboration, M. Guagnelli, R. Petronzio, J. Rolf, S. Sint, R. Sommer and $\mathrm{U}$. Wolff, Non-perturbative results for the coefficients $b_{\mathrm{m}}$ and $b_{\mathrm{A}}-b_{\mathrm{P}}$ in $O(a)$ improved lattice QCD, Nucl. Phys. B595 (2001) 44 [hep-lat/0009021].

[9] ALPHA Collaboration, J. Heitger and J. Wennekers, Effective heavy-light meson energies in small-volume quenched QCD, J. High Energy Phys. 02 (2004) 064 [hep-lat/0312016].

[10] M. Lüscher, R. Narayanan, P. Weisz and U. Wolff, The Schrödinger functional: A renormalizable probe for non-abelian gauge theories, Nucl. Phys. B384 (1992) 168 [hep-lat/9207009].

[11] S. Sint, On the Schrödinger functional in QCD, Nucl. Phys. B421 (1994) 135 [hep-lat/9312079].

[12] S. Sint, One-loop renormalization of the QCD Schrödinger functional, Nucl. Phys. B451 (1995) 416 [hep-lat/9504005].

[13] K. Jansen et. al., Non-perturbative renormalization of lattice QCD at all scales, Phys. Lett. B372 (1996) 275 [hep-lat/9512009].

[14] ALPHA Collaboration, J. Heitger and R. Sommer, Non-perturbative heavy quark effective theory, J. High Energy Phys. 02 (2004) 022 [hep-lat/0310035].

[15] M. Guagnelli, F. Palombi, R. Petronzio and N. Tantalo, $F_{\mathrm{B}}$ and two scales problems in lattice QCD, Phys. Lett. B546 (2002) 237 [hep-lat/0206023].

[16] D. Guazzini, R. Sommer and N. Tantalo, Precision for B-meson matrix elements, J. High Energy Phys. 01 (2008) 076 [0710.2229].

[17] ALPHA Collaboration, J. Rolf and S. Sint, A precise determination of the charm quark's mass in quenched QCD, J. High Energy Phys. 12 (2002) 007 [hep-ph/0209255].

[18] ALPHA Collaboration, J. Heitger and A. Jüttner, Lattice cutoff effects for $f_{\mathrm{D}_{\mathrm{s}}}$ with improved Wilson fermions - a final lesson from the quenched case, J. High Energy Phys. 05 (2009) 101 [0812.2200]. 
[19] ALPHA Collaboration, G. von Hippel, R. Sommer, J. Heitger, S. Schaefer and N. Tantalo, $\mathrm{D}_{\mathrm{s}}$ physics from fine lattices, PoS LATTICE2008 (2008) 227 [0810.0214].

[20] ALPHA Collaboration, M. Della Morte, R. Hoffmann and R. Sommer, Non-perturbative improvement of the axial current for dynamical Wilson fermions, J. High Energy Phys. 03 (2005) 029 [hep-lat/0503003].

[21] T. Bhattacharya, R. Gupta, W.-J. Lee, S. R. Sharpe and J. M. S. Wu, Improved bilinears in lattice QCD with non-degenerate quarks, Phys. Rev. D73 (2006) 034504 [hep-lat/0511014].

[22] T. Bhattacharya, R. Gupta, W.-J. Lee and S. R. Sharpe, $O(a)$ improved renormalization constants, Phys. Rev. D63 (2001) 074505 [hep-lat/0009038].

[23] R. Sommer, Non-perturbative QCD: renormalization, $O(a)$-improvement and matching to Heavy Quark Effective Theory, Lectures given at ILFTN Workshop on "Perspectives in Lattice QCD", Nara, Japan, 31 October - 11 November 2005 [hep-lat/0611020].

[24] M. Lüscher, S. Sint, R. Sommer and P. Weisz, Chiral symmetry and $O(a)$ improvement in lattice QCD, Nucl. Phys. B478 (1996) 365 [hep-lat/9605038].

[25] ALPHA Collaboration, M. Lüscher, S. Sint, R. Sommer, P. Weisz and U. Wolff, Non-perturbative O(a) improvement of lattice QCD, Nucl. Phys. B491 (1997) 323 [hep-lat/9609035].

[26] ALPHA Collaboration, M. Della Morte, R. Sommer and S. Takeda, On cutoff effects in lattice QCD from short to long distances, Phys. Lett. B672 (2009) 407 [0807.1120].

[27] ALPHA Collaboration, M. Della Morte, P. Fritzsch, J. Heitger, H. B. Meyer, H. Simma and R. Sommer, Towards a non-perturbative matching of HQET and QCD with dynamical light quarks, PoS LAT2007 (2007) 246 [0710.1188].

[28] ALPHA Collaboration, M. Della Morte, P. Fritzsch, J. Heitger and R. Sommer, Non-perturbative quark mass dependence in the heavy-light sector of two-flavour QCD, PoS LATTICE2008 (2008) 226 [0810.3166].

[29] L. Del Debbio, L. Giusti, M. Lüscher, R. Petronzio and N. Tantalo, QCD with light Wilson quarks on fine lattices (I): first experiences and physics results, J. High Energy Phys. 02 (2007) 056 [hep-lat/0610059].

[30] ALPHA Collaboration, M. Della Morte, P. Fritzsch, B. Leder, H. B. Meyer, H. Simma, R. Sommer, S. Takeda, O. Witzel and U. Wolff, Preparing for $N_{\mathrm{f}}=2$ simulations at small lattice spacings, PoS LAT2007 (2007) 255 [0710.1263].

[31] M. Hasenbusch, Speeding up the Hybrid-Monte-Carlo algorithm for dynamical fermions, Phys. Lett. B519 (2001) 177 [hep-lat/0107019].

[32] M. Hasenbusch and K. Jansen, Speeding up lattice QCD simulations with clover-improved Wilson fermions, Nucl. Phys. B659 (2003) 299 [hep-lat/0211042].

[33] ALPHA Collaboration, M. Della Morte, F. Knechtli, J. Rolf, R. Sommer, I. Wetzorke and U. Wolff, Simulating the Schrödinger functional with two pseudo-fermions, Comput. Phys. Commun. 156 (2003) 62 [hep-lat/0307008].

[34] ALPHA Collaboration, H. B. Meyer, H. Simma, R. Sommer, M. Della Morte, O. Witzel and U. Wolff, Exploring the HMC trajectory-length dependence of autocorrelation times in lattice QCD, Comput. Phys. Commun. 176 (2007) 91 [hep-lat/0606004]. 
[35] ALPHA Collaboration, U. Wolff, Monte Carlo errors with less errors, Comput. Phys. Commun. 156 (2004) 143 [hep-lat/0306017].

[36] ALPHA Collaboration, S. Sint and P. Weisz, Further results on $O(a)$ improved lattice QCD to one-loop order of perturbation theory, Nucl. Phys. B502 (1997) 251 [hep-lat/9704001].

[37] ALPHA Collaboration, M. Della Morte, R. Hoffmann, F. Knechtli and U. Wolff, Impact of large cutoff-effects on algorithms for improved Wilson fermions, Comput. Phys. Commun. 165 (2005) 49 [hep-lat/0405017].

[38] L. D. Debbio, L. Giusti, M. Lüscher, R. Petronzio and N. Tantalo, Stability of lattice QCD simulations and the thermodynamic limit, J. High Energy Phys. 02 (2006) 011 [hep-lat/0512021].

[39] ALPHA Collaboration, M. Della Morte, R. Frezzotti, J. Heitger, J. Rolf, R. Sommer and U. Wolff, Computation of the strong coupling in QCD with two dynamical flavours, Nucl. Phys. B713 (2005) 378 [hep-lat/0411025].

[40] ALPHA Collaboration, M. Della Morte, R. Hoffmann, F. Knechtli, J. Rolf, R. Sommer, I. Wetzorke and U. Wolff, Non-perturbative quark mass renormalization in two-flavor QCD, Nucl. Phys. B729 (2005) 117 [hep-lat/0507035].

[41] ALPHA Collaboration, M. Della Morte, N. Garron, M. Papinutto and R. Sommer, Heavy quark effective theory computation of the mass of the bottom quark, J. High Energy Phys. 01 (2007) 007 [hep-ph/0609294].

[42] ALPHA Collaboration, B. Blossier, M. Della Morte, N. Garron and R. Sommer, HQET at order 1/m: I. Non-perturbative parameters in the quenched approximation, 1001.4783.

[43] ALPHA Collaboration, J. Heitger, A. Jüttner, R. Sommer and J. Wennekers, Non-perturbative tests of heavy quark effective theory, J. High Energy Phys. 11 (2004) 048 [hep-ph/0407227].

[44] P. Fritzsch, B-meson properties from non-perturbative matching of HQET to finite-volume two-flavour QCD, Ph.D. Thesis (2009). Universität Münster. 